\title{
Measurement of the mass of the $\tau$ lepton
}

J. Z. Bai, ${ }^{1}$ O. Bardon,${ }^{6}$ R. A. Becker-Szendy ${ }^{8}$ I. Blum, ${ }^{11}$ A. Breakstone,${ }^{9}$ T. Burnett,${ }^{12}$ G. P. Chen, ${ }^{1}$ H. F. Chen, ${ }^{4}$ J. Chen, ${ }^{5}$ S. J. Chen, ${ }^{1}$ S. M. Chen, ${ }^{1}$ Y. Chen, ${ }^{1}$ Y. B. Chen, ${ }^{1}$ Y. Q. Chen, ${ }^{1}$ B. S. Cheng, ${ }^{1}$ R. F. Cowan, ${ }^{6}$ H. C. Cui ${ }^{1}$ X. Z. Cui, ${ }^{1}$ H. L. Ding, ${ }^{1}$ Z. Z. Du, ${ }^{1}$ W. Dunwoodie, ${ }^{8}$ X. L. Fan, ${ }^{1}$ J. Fang, ${ }^{1}$ C. S. Gao, ${ }^{1}$ M. L. Gao, ${ }^{1}$ S. Q. Gao, ${ }^{1}$

W. X. Gao, ${ }^{1}$ P. Gratton, ${ }^{11}$ J. H. Gu, ${ }^{1}$ S. D. Gu, ${ }^{1}$ W. X. Gu, ${ }^{1}$ Y. F. Gu, ${ }^{1}$ Y. N. Guo, ${ }^{1}$ S. W. Han, ${ }^{1}$ Y. Han,${ }^{1}$

F. A. Harris, ${ }^{9}$ M. Hatanaka, ${ }^{3}$ J. He,${ }^{1}$ K. R. He, ${ }^{1}$ M. He,${ }^{7}$ D. G. Hitlin,${ }^{3}$ G. Y. $\mathrm{Hu},{ }^{1}$ T. Hu, ${ }^{1}$ X. Q. Hu, ${ }^{1}$

D. Q. Huang, ${ }^{1}$ Y. Z. Huang, ${ }^{1}$ J. M. Izen, ${ }^{11}$ Q. P. Jia,${ }^{5}$ C. H. Jiang, ${ }^{1}$ Z. Z. Jiang, ${ }^{7}$ S. Jin,${ }^{1}$ Y. Jin, ${ }^{1}$ L. Jones, ${ }^{3}$

S. H. Kang, ${ }^{1}$ Z. J. Ke, ${ }^{1}$ M. H. Kelsey, ${ }^{3}$ B. K. Kim, ${ }^{11}$ Y. F. Lai, ${ }^{1}$ H. B. Lan, ${ }^{1}$ P. F. Lang, ${ }^{1}$ A. Lankford,${ }^{10}$ F. Li,${ }^{1}$ J. $\mathrm{Li}^{1}{ }^{1}$ P. Q. Li, ${ }^{1}$ Q. Li, ${ }^{7}$ R. B. Li, ${ }^{1}$ W. $\mathrm{Li}^{1}{ }^{1}$ W. D. $\mathrm{Li},{ }^{1}$ W. G. Li, ${ }^{1}$ X. H. Li, ${ }^{1}$ X. N. Li, ${ }^{1}$ Y. S. Li, ${ }^{1}$ S. Z. Lin, ${ }^{1}$

H. M. Liu ${ }^{1}{ }^{J}$. Liu, ${ }^{1}$ J. H. Liu, ${ }^{1}$ Q. Liu,${ }^{1}$ R. G. Liu ${ }^{1}$ Y. Liu, ${ }^{1}$ Z. A. Liu, ${ }^{1}$ X. C. Lou, ${ }^{11}$ B. Lowery,${ }^{11, *}$ F. Lu, ${ }^{1}$

J. G. Lu, ${ }^{1}$ Y. Luo, ${ }^{1}$ A. M. Ma, ${ }^{1}$ D. H. Ma, ${ }^{1}$ E. C. Ma, ${ }^{1}$ J. M. Ma, ${ }^{1}$ H. S. Mao, ${ }^{1}$ Z. P. Mao, ${ }^{1}$ R. Malchow, ${ }^{5}$

M. Mandelkern,${ }^{10}$ H. Marsiske,${ }^{8}$ X. C. Meng, ${ }^{1}$ H. L. Ni,${ }^{1}$ J. Nie,${ }^{1}$ S. L. Olsen, ${ }^{9}$ J. Oyang, ${ }^{3}$ D. Paluselli, ${ }^{9}$ L. J. Pan, ${ }^{9}$ J. Panetta, ${ }^{3}$ F. Porter, ${ }^{3}$ E. Prabhakar, ${ }^{3}$ N. D. Qi,${ }^{1}$ Y. K. Que, ${ }^{1}$ J. Quigley, ${ }^{6}$ G. Rong, ${ }^{1}$ M. Schernau, ${ }^{10}$ B. Schmid, ${ }^{10}$ J. Schultz, ${ }^{10}$ Y. Y. Shao, ${ }^{1}$ B. W. Shen,${ }^{1}$ D. L. Shen, ${ }^{1}$ H. Shen,${ }^{1}$ X. Y. Shen ${ }^{1}$ H. Y. Sheng, ${ }^{1}$ H. Z. Shi, ${ }^{1}$ X. R. Shi, ${ }^{3}$ A. Smith, ${ }^{10}$ E. Soderstrom, ${ }^{8}$ X. F. Song, ${ }^{1}$ J. Standifird, ${ }^{11}$ D. Stoker,${ }^{10}$ F. Sun, ${ }^{1}$ H. S. Sun, ${ }^{1}$ S. J. Sun, ${ }^{1}$

J. Synodinos, ${ }^{8}$ Y. P. Tan, ${ }^{1}$ S. Q. Tang, ${ }^{1}$ W. Toki ${ }^{5}$ G. L. Tong, ${ }_{1}^{1}$ E. Torrence,${ }^{6}$ F. Wang, ${ }_{1}^{1}$ L. S. Wang, ${ }^{1}$ L. Z. Wang, ${ }^{1}$ M. Wang, ${ }^{1}$ P. Wang, ${ }^{1}$ P. L. Wang, ${ }^{1}$ S. M. Wang, ${ }^{1}$ T. J. Wang, ${ }^{1}$ Y. Y. Wang, ${ }^{1}$ C. L. Wei, ${ }^{1}$ S. Whittaker, ${ }^{2}$ R. Wilson, ${ }^{5}$

W. J. Wisniewski, ${ }^{13,+}$ Y. G. Wu, ${ }^{1}$ D. M. Xi,${ }^{1}$ X. M. Xia, ${ }^{1}$ P. P. Xie,${ }^{1}$ X. X. Xie, ${ }^{1}$ W. J. Xiong, ${ }^{1}$ D. Z. Xu,${ }^{1}$

M. K. Xu, ${ }^{14}$ R. S. Xu, ${ }^{1}$ Y. D. Xu, ${ }^{14}$ Z. Q. Xu, ${ }^{1}$ S. T. Xue, ${ }^{1}$ R. Yamamoto, ${ }^{6}$ J. Yan, ${ }^{1}$ W. G. Yan, ${ }^{1}$ C. M. Yang, ${ }^{1}$

C. Y. Yang, ${ }^{1}$ J. Yang, ${ }^{1}$ W. Yang, ${ }^{1}$ H. B. Yao, ${ }^{1}$ M. H. Ye,${ }^{1}$ S. W. Ye,${ }^{4}$ S. Z. Ye ${ }^{1}$ C. S. Yu, ${ }^{1}$ C. X. Yu, ${ }^{1}$ Y. H. Yu, ${ }^{14}$

Z. Q. Yu, ${ }_{1}^{1}$ C. Z. Yuan, ${ }^{1}$ J. Y. Zeng, ${ }^{1}$ B. Y. Zhang, ${ }^{1}$ C. C. Zhang, ${ }^{1}$ D. H. Zhang, ${ }^{1}$ H. L. Zhang, ${ }^{1}$ J. Zhang, ${ }^{1}$

J. W. Zhang, ${ }^{1}$ L. S. Zhang, ${ }^{1}$ S. Q. Zhang, ${ }^{1}$ Y. Zhang, ${ }^{1}$ Y. Y. Zhang, ${ }^{1}$ D. X. Zhao, ${ }_{1}^{1}$ H. W. Zhao, ${ }^{1}$ J. W. Zhao, ${ }^{1}$ M. Zhao, ${ }^{1}$ P. D. Zhao, ${ }^{1}$ W. R. Zhao, ${ }^{1}$ J. P. Zheng, ${ }^{1}$ L. S. Zheng, ${ }^{1}$ Z. P. Zheng, ${ }^{1}$ G. P. Zhou, ${ }^{1}$ H. S. Zhou, ${ }^{1}$ Li Zhou, ${ }^{1}$ X. F. Zhou, ${ }^{1}$ Y. H. Zhou, ${ }^{1}$ H. G. Zhu, ${ }^{1}$ Q. M. Zhu, ${ }^{1}$ Y. C. Zhu, ${ }^{1}$ Y. S. Zhu, ${ }^{1}$ B. A. Zhuang, ${ }^{1}$ G. Zioulas ${ }^{10}$

(BES Collaboration)

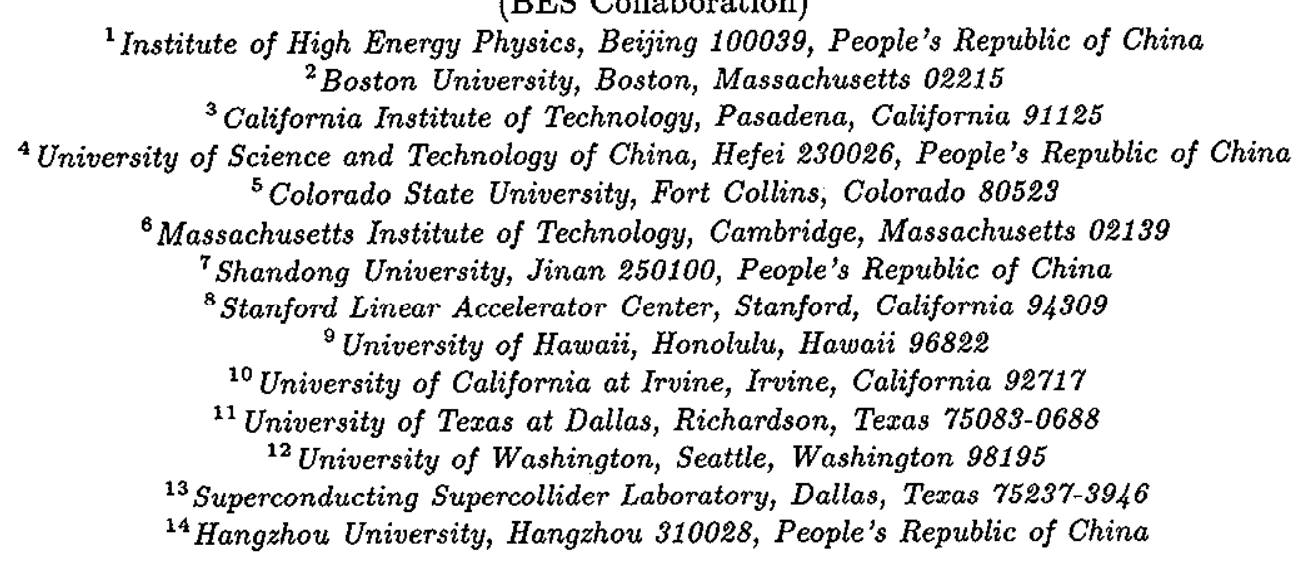

(Received 25 July 1995)

\begin{abstract}
A data-driven energy scan in the immediate vicinity of the $\tau$ pair production threshold has been performed using the Beijing Spectrometer at the Beijing Electron-Positron Collider. Approximately $5 \mathrm{pb}^{-1}$ of data, distributed over 12 scan points, have been collected. A previous mass value for the $\tau$ lepton, obtained using only the $e \mu$ final state, has been published. In this paper, the final BES result on the mass measurement is presented. The analysis is based on the combined data from the $e e, e \mu, e h, \mu \mu, \mu h$, and $h h$ final states, where $h$ denotes a charged $\pi$ or $K$. A maximum likelihood fit to the $\tau$ pair production cross section data yields the value $m_{\tau}=1776.96_{-0.21}^{+0.18+0.25} \mathrm{MeV}$.
\end{abstract}

PACS number(s): $14.60 . \mathrm{Fg}, 13.10 .+\mathrm{q}$

\section{INTRODUCTION}

\footnotetext{
*Present address: Kansas State University, Manhattan, Kansas 66506-2601.

${ }^{\dagger}$ Present address: Stanford Linear Accelerator Center, Stanford, California 94309 .
}

Measurements of the mass, lifetime, and electronic branching fraction of the $\tau$ lepton have been improved to the extent that they can be used to provide a significant test of lepton universality. The most precise measure- 
ment to date of the mass of the $\tau$ lepton is described in this paper.

As the third of the sequential charged leptons, the $\tau$ decays similarly to the $\mu$ by virtue of its coupling to a virtual $W$ boson and a neutrino; however, the $\tau$ has many more open decay channels as a consequence of its large mass. Within the standard model, leptonic decay rates are given by [1]

$$
\Gamma\left(L \rightarrow l \nu_{L} \bar{\nu}_{l}\right)=\frac{G_{L}^{2} m_{L}^{5}}{192 \pi^{3}} F_{\operatorname{cor}}\left(m_{L}, m_{l}\right)
$$

where $m_{L}$ is the mass of the parent lepton $L, m_{l}$ is the mass of the daughter lepton $l, G_{L}$ is the Fermi weak coupling constant, and the correction factor $F_{\text {cor }}$ is given by

$$
F_{\mathrm{cor}}=f\left(\frac{m_{l}^{2}}{m_{L}^{2}}\right) F_{W} F_{\mathrm{rad}}
$$

with

$$
\begin{gathered}
f(x)=1-8 x+8 x^{3}-x^{4}-12 x^{2} \ln x, \\
F_{W}=\left[1+\frac{3}{5} \frac{m_{L}^{2}}{m_{W}^{2}}\right],
\end{gathered}
$$

and

$$
F_{\mathrm{rad}}=\left[1+\frac{\alpha\left(m_{L}\right)}{2 \pi}\left(\frac{25}{4}-\pi^{2}\right)\right]
$$

The function $f(x)$ results from the integration of the squared matrix element for $\tau$ decay over the three-body final state phase space. The correction factor $F_{W}$ accounts for the nonlocal structure of the $W$ propagator, where $m_{W}$ is the mass of the $W$ boson; the factor $F_{\text {rad }}$ arises due to initial and final state radiative corrections $\left\{\alpha\left(m_{\tau}\right)^{-1}=133.3\right.$ [1]]. It should be noted that the current value of the Fermi weak coupling constant, $G_{F}=1.16639(2) \times 10^{-5} \mathrm{GeV}^{-2}$, is obtained from Eqs. (1)-(5) in the case of $\mu$ decay by inserting the values $m_{\mu}=105.658389 \pm 0.000034 \mathrm{MeV}, \Gamma(\mu \rightarrow e \nu \bar{\nu})^{-1}=$ $(2.19703 \pm 0.00004) \times 10^{-6} \mathrm{~s}, m_{W}=80.22 \pm 0.26 \mathrm{GeV}$ [2], with $\alpha\left(m_{\mu}\right)^{-1}=136$ [1].

The electronic decays of the $\tau$ and muon can be related through Eq. (1) where the substitutions $\Gamma(\tau \rightarrow e \nu \tilde{\nu})=$ $B_{\tau \rightarrow e \nu \bar{\nu}} / t_{\tau}$ and $\Gamma(\mu \rightarrow e \nu \bar{\nu})=1 / t_{\mu}$ yield

$$
\left(\frac{G_{\tau \rightarrow e \nu \bar{\nu}}}{G_{\mu \rightarrow e \nu \bar{\nu}}}\right)^{2}=\left(\frac{m_{\mu}}{m_{\tau}}\right)^{5}\left(\frac{t_{\mu}}{t_{\tau}}\right) B_{\tau \rightarrow e \nu \bar{\nu}} \frac{F_{\text {cor }}\left(m_{\mu}, m_{e}\right)}{F_{\text {cor }}\left(m_{\tau}, m_{e}\right)}
$$

where $t_{l}$ denotes the lepton lifetime, and $B_{\tau \rightarrow e \nu \bar{\nu}}$ the branching fraction for the decay $\tau \rightarrow e \nu \bar{\nu}$; the functions $F_{\text {cor }}$, whose contributions are listed in Table I, together contribute to the ratio of the squared coupling constants at the level of 0.0004 . The value of this ratio is unity under the assumption of lepton universality. Prior to the
TABLE I. The corrections to the $\mu$ and $\tau$ decay rates, calculated using $\alpha\left(m_{\mu}\right)^{-1}=136$ and $\alpha\left(m_{\tau}\right)^{-1}=133.3[1]$.

\begin{tabular}{lr}
\hline Correction & Value \\
\hline$f\left(m_{e}^{2} / m_{\mu}^{2}\right)$ & 0.9998 \\
$F_{W}\left(m_{\mu}\right)$ & 1.0000 \\
$F_{\text {rad }}\left(m_{\mu}\right)$ & 0.9958 \\
$f\left(m_{e}^{2} / m_{\tau}^{2}\right)$ & 1.0000 \\
$F_{W}\left(m_{\tau}\right)$ & 1.0003 \\
$F_{\text {rad }}\left(m_{\tau}\right)$ & 0.9957 \\
\hline$F_{\text {cor }}\left(m_{\mu}, m_{e}\right)$ & 0.99558 \\
$F_{\text {cor }}\left(m_{\tau}, m_{e}\right)$ & 0.99597 \\
\hline \hline
\end{tabular}

present experiment, the uncertainty in the value of $m_{\tau}$ was 3-4 MeV. Since $m_{\tau}$ enters Eq. (6) at the fifth power, lepton universality could be tested only at the $1 \%$ level at best. The goal of this experiment was to improve the measurement precision of $m_{\tau}$ by an order of magnitude, thus making possible tests of universality down to the $0.1 \%$ level.

The experimental procedure has been described previously [3]. A data-driven search near threshold for $e^{+} e^{-} \rightarrow \tau^{+} \tau^{-}$was performed in which candidate events were identified by requiring that one $\tau$ decay via $\tau \rightarrow e \nu \bar{\nu}$, and the other via $\tau \rightarrow \mu \nu \bar{\nu}$. The $\tau$ lepton mass value, obtained from a fit to the energy dependence of the resulting $\tau^{+} \tau^{-}$cross section data, was $m_{\tau}=1776.9_{-0.5}^{+0.4} \pm 0.2 \mathrm{MeV}$ [3].

In this paper the analysis is extended to include the $e e, \mu \mu, e h, \mu h$, and $h h$ final states, where $h$ can be either a charged $\pi$ or $K$. These final states provide additional information which is independent of the $e \mu$ events which drove the energy scan, and result in a reduction in the statistical uncertainty in the mass value by a factor of 2 .

\section{THE BEIJING COLLIDER AND BEIJING SPECTROMETER}

The Beijing Electron Positron Collider (BEPC) [4], shown schematically in Fig. 1, operates in the $3-5 \mathrm{GeV}$

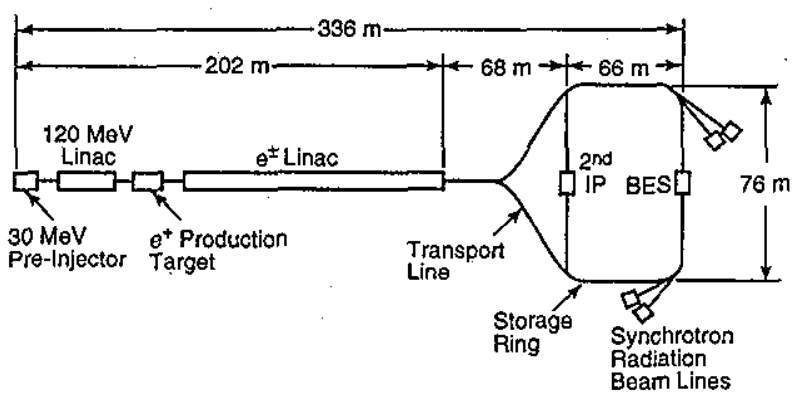

FIG. 1. The Beijing Electron Positron Collider (BEPC). The $202 \mathrm{~m}$ injection linac leading to the $240 \mathrm{~m}$ circumference storage ring is shown at the left. The electrons circulate clockwise and the positrons counterclockwise. The BES sits on the side of the ring opposite the injection linac. 
c.m. energy range. Near $\tau^{+} \tau^{-}$threshold, the peak luminosity is $5 \times 10^{30} \mathrm{~cm}^{-2} \mathrm{~s}^{-1}$, the luminosity-weighted uncertainty in the mean c.m. energy is $\sim 0.1 \mathrm{MeV}$, and the distribution of c.m. energy about its nominal value is described by a Gaussian with standard deviataion $\sim 1.4 \mathrm{MeV}$. The absolute energy scale and energy spread are determined by linear interpolation between the results of repeated scans of the $J / \psi$ and $\psi^{\prime}[\psi(2 S)]$. resonances.

The Beijing Spectrometer (BES), shown in Fig. 2, is a conventional cylindrical detector described in detail in Ref. [5]. A four-layer central drift chamber (CDC) surrounding the beam pipe provides trigger information. Charged tracks are reconstructed in a 40-layer main drift chamber (MDC) which provides solid angle coverage of $85 \%$ of $4 \pi$. Momentum resolution of $2.1 \% \sqrt{1+p^{2}}(p$ in $\mathrm{GeV} / c)$ and energy loss $(d E / d x)$ resolutions of $8.5 \%$ for electrons, $9.4 \%$ for muons, and $11 \%$ for hadrons are obtained in the present experiment. Scintillation counters provide time-of-flight (TOF) measurements over $76 \%$ of $4 \pi$, with resolutions of $390 \mathrm{ps}$ for electrons, $410 \mathrm{ps}$ for muons, and $450 \mathrm{ps}$ for hadrons. A 12-radiationlength, lead-gas barrel shower counter (BSC), operating in limited streamer mode, measures the energies of elec- trons and photons over $80 \%$ of the total solid angle, and achieves energy resolution $\sigma_{E} / E=0.22 / \sqrt{E}(E$ in $\mathrm{GeV})$ for electrons, and spatial resolutions $\sigma_{\phi}=4.5 \mathrm{mrad}$ and $\sigma_{z}=2 \mathrm{~cm}$. A solenoidal magnet provides a $0.4 \mathrm{~T}$ magnetic field in the central tracking region of the detector. Three double-layer muon counters instrument the magnet flux return, and serve to identify muons of momentum greater than $500 \mathrm{MeV} / c$. They cover $68 \%$ of the total solid angle with longitudinal (transverse) spatial resolution $5 \mathrm{~cm}(3 \mathrm{~cm})$. End-cap time-of-flight and shower counters extend coverage to the forward and backward regions.

\section{III. $\tau^{+} \tau^{-}$PRODUCTION CROSS SECTION NEAR THRESHOLD}

The likelihood function used to estimate the $\tau$ lepton mass value incorporates the $\tau^{+} \tau^{-}$production cross section near threshold [6]. Including the c.m. energy spread $\Delta$, initial state radiation corrections $[7] F(x, W)$, and vacuum polarization corrections [8] $\Pi(W)$, the cross section is

$$
\sigma\left(W, m_{\tau}\right)=\frac{1}{\sqrt{2 \pi} \Delta} \int_{2 m_{\tau}}^{\infty} d W^{\prime} \exp \left(\frac{-\left(W-W^{\prime}\right)^{2}}{2 \Delta^{2}}\right) \int_{0}^{1-\frac{4 m^{2}}{W^{\prime 2}}} d x F\left(x, W^{\prime}\right) \sigma_{1}\left(W^{\prime} \sqrt{1-x}, m_{\tau}\right)
$$

where $\sigma_{1}$ is given by

$$
\sigma_{1}\left(W, m_{\tau}\right)=\frac{4 \pi \alpha^{2}}{3 W^{2}} \frac{\beta\left(3-\beta^{2}\right)}{2} \frac{F_{c}(\beta) F_{r}(\beta)}{[1-\Pi(W)]^{2}}
$$

$W$ is the c.m. energy, and $\beta=\sqrt{1-\left(\frac{2 m_{x}}{W}\right)^{2}}$. The Coulomb interaction and final state radiation corrections are described by the functions $F_{c}(\beta)$ and $F_{r}(\beta)[9]$. The effect of these corrections on the lowest order QED cross section is shown in Fig. 3.

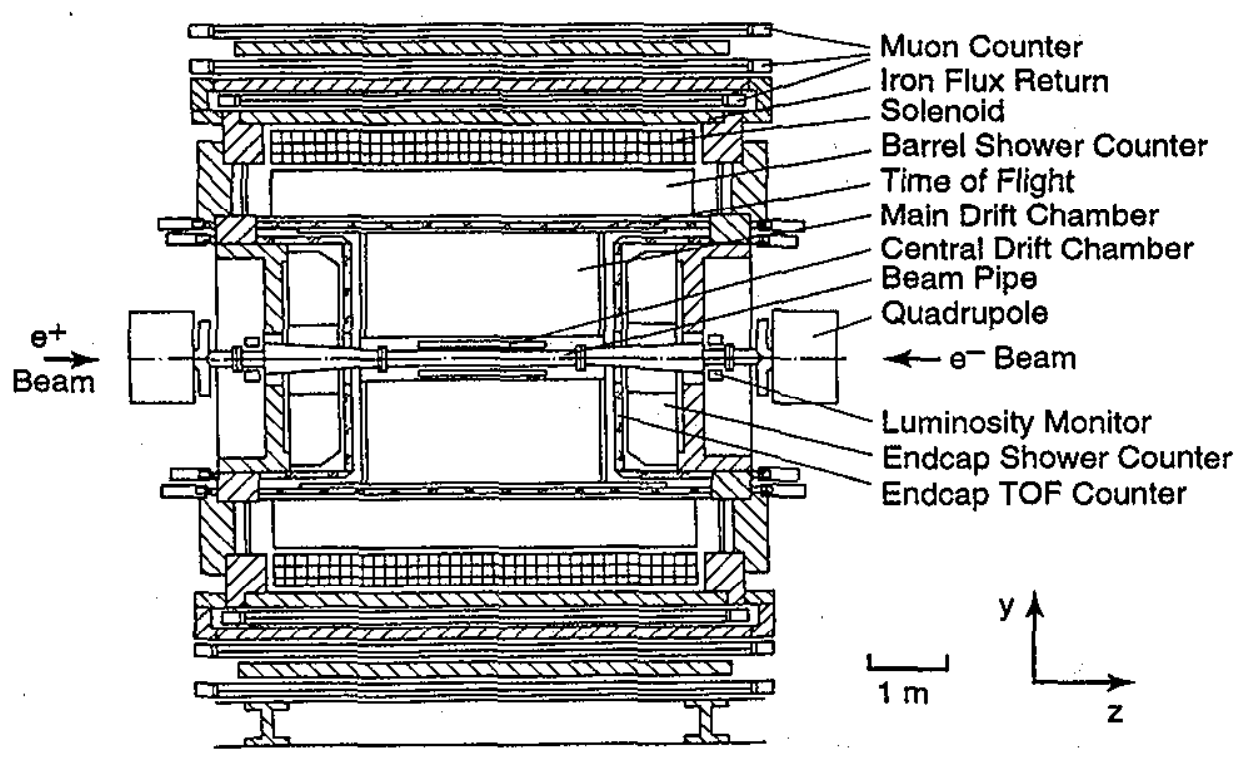

FIG. 2. The Beijing Spectrometer (BES) in $y$ - $z$ projection. 


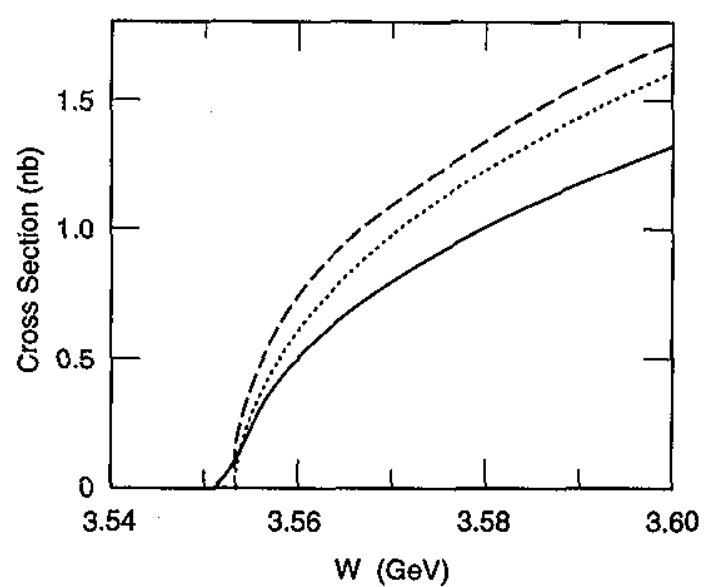

FIG. 3. The $\tau^{+} \tau^{-}$production cross section near threshold as a function of c.m. energy $W$. The dotted curve shows the lowest order QED cross section, the dashed curve takes into account the Coulomb interaction and final state radiation, and the solid curve shows the final cross section used in this analysis after initial state radiation, vacuum polarization, and beam energy spread have been taken into account. Note that the beam energy spread and the initial state radiation correction smear out the sharp step at threshold caused by the Coulomb interaction. The curves are calculated using $m_{r}=1776.9 \mathrm{MeV}[3]$.

\section{IV. c.m. ENERGY SCAN}

A total of $5 \mathrm{pb}^{-1}$ of $e^{+} e^{-}$collision data were collected near $\tau^{+} \tau^{-}$threshold over a 2 month period beginning in November 1991. The range of c.m. energy in which the $\tau^{+} \tau^{-}$cross section is most sensitive to the $\tau$ mass is of the order of the beam energy spread around $\tau^{+} \tau^{-}$ threshold; it was important, therefore, to devise a running strategy which would locate the $\tau^{+} \tau^{-}$threshold region and maximize the integrated luminosity there. To accomplish this, the beam energy was set initially to approximately $1784.1 \mathrm{MeV}$, which was the average value of the mass of the $\tau$ lepton at that time [10]. During the run, the data were searched for events in which one $\tau$ decayed via $e \nu \bar{\nu}$ and the other via $\mu \nu \bar{\nu}$. Such so-called $e \mu$ events provide a very clean signature for $\tau$ pair production; indeed, it was this signature which led to the discovery of the $\tau$ lepton [11].

After each $250-400 \mathrm{nb}^{-1}$ of integrated luminosity, a new estimate of the mass was made based on the number and distribution in c.m. energy of the $e \mu$ events observed in all of the data accumulated to that point [3]; in this way a new prediction of the most sensitive c.m. energy at which to run was obtained. The c.m. energy was changed to the new value only if the difference was greater than $0.4 \mathrm{MeV}$. Following this strategy, an integrated luminosity of $\sim 4.3 \mathrm{pb}^{-1}$ was accumulated at ten c.m. energy values within a range of $24 \mathrm{MeV}$ around $\tau^{+} \tau^{-}$threshold.

The sequence of energies is shown in Fig. 4, and the corresponding data [12] are summarized in Table II. The ten-step search yielded seven $e \mu$ events. The 11 th and

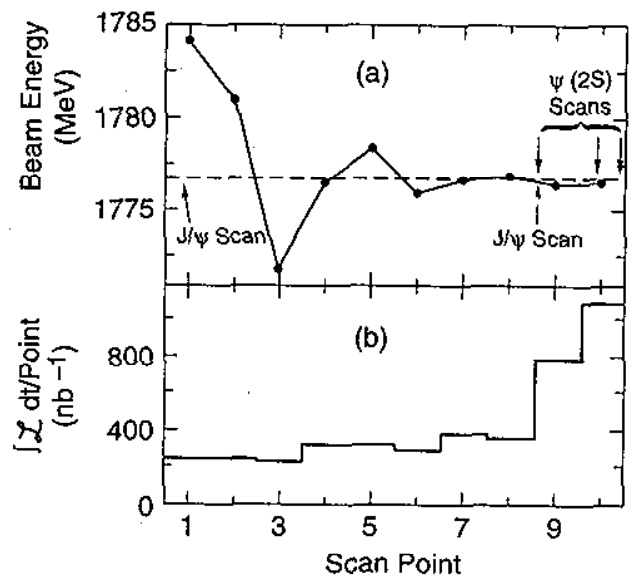

FIG. 4. (a) The variation of the beam energy value with scan point, showing the convergence to $\tau^{+} \tau^{-}$production threshold. The $J / \psi$ and $\psi^{\prime}$ resonance scans (Tables VIII and IX, and Fig. 19) were performed in the sequence indicated. (b) The integrated luminosity accumulated at each scan point. The luminosity per scan point is approximately constant for the first eight points, then increases significantly for the last two points. This is because the likelihood fit indicates that a change in beam energy is required less frequently as threshold is approached.

12th points in Table II were taken well above threshold, where the cross section is relatively large and slowly varying with c.m. energy, in order to provide an improved estimate of the absolute detection efficiency (see Sec. VIII). The mass value obtained from a fit to the energy dependence of the $\tau^{+} \tau^{-}$cross section was $m_{\tau}=$ $1776.9_{-0.5}^{+0.4} \pm 0.2 \mathrm{MeV}[3]$.

\section{EXTENDED ANAEYSIS}

A more general analysis of the data, which uses a simplified scheme of event selection and particle identifica-

TABLE II. A chronological summary of the $\tau^{+} \tau^{-}$threshold scan data; $W$ denotes the corrected c.m. energy, $\Delta$ the spread in c.m. energy [12] [see Eq. (6)], and $\mathcal{L}$ the integrated luminosity.

\begin{tabular}{ccccc}
\hline \hline Scan point & $\begin{array}{c}W / 2 \\
(\mathrm{MeV})\end{array}$ & $\begin{array}{c}\Delta \\
(\mathrm{MeV})\end{array}$ & $\begin{array}{c}\mathcal{L} \\
\left(\mathrm{nb}^{-1}\right)\end{array}$ & $\begin{array}{c}N \\
(e \mu \text { events })\end{array}$ \\
\hline 1 & 1784.19 & 1.34 & 245.8 & 2 \\
2 & 1780.99 & 1.33 & 248.9 & 1 \\
3 & 1772.09 & 1.36 & 232.8 & 0 \\
4 & 1776.57 & 1.37 & 323.0 & 0 \\
5 & 1778.49 & 1.44 & 322.5 & 2 \\
6 & 1775.95 & 1.43 & 296.9 & 0 \\
7 & 1776.75 & 1.47 & 384.0 & 0 \\
8 & 1776.98 & 1.47 & 360.8 & 1 \\
9 & 1776.45 & 1.44 & 794.1 & 0 \\
10 & 1776.62 & 1.40 & 1109.1 & 1 \\
11 & 1799.51 & 1.44 & 499.7 & 5 \\
12 & 1789.55 & 1.43 & 250.0 & 2 \\
\hline \hline
\end{tabular}


tion, is presented in this paper. This second analysis incorporates the $e \mu$ final state and several additional twoprong $\tau^{+} \tau^{-}$final states, and results in a reduction of the statistical error in the mass value of the $\tau$ lepton to the level of the systematic uncertainty.

To select candidate two-prong $\tau^{+} \tau^{-}$decay events from the $11.7 \times 10^{6}$ triggers representing the data listed in Table II, it is first required that exactly two charged tracks be well reconstructed, without regard to net charge. For each track, the point of closest approach to the beam line must have radius $\leq 1.5 \mathrm{~cm}$ and $|z| \leq 15 \mathrm{~cm}$ where $z$ is measured along the beam line from the nominal beam crossing point; in addition $\left|z_{1}-z_{2}\right|$ must be less than $5 \mathrm{~cm}$. Furthermore, each track is required to satisfy $|\cos \theta| \leq 0.75$, where $\theta$ is the polar angle, to ensure that it is contained within the barrel region of the detector. These criteria reduce the data sample by a factor of $\sim 20$.

Next, it is required that the transverse momentum of each charged track be above the $100 \mathrm{MeV} / c$ minimum needed to traverse the barrel time-of-flight counter and reach the outer radius of the barrel shower counter in the $0.4 \mathrm{~T}$ axial magnetic field. In addition, the magnitude of the momentum must be less than the maximum expected in any $\tau$ decay at the given c.m. energy within a tolerance of three standard deviations in momentum resolution. These constraints on momentum reduce the data sample by over an order of magnitude, leaving $\sim 40000$ events. Most of this reduction is due to the removal of Bhabha scattering and $\mu$ pair production events.

The search for $\tau^{+} \tau^{-}$production events is restricted to final states which do not contain $\pi^{0}$ 's or $\gamma^{\prime}$ s. Consequently, a further requirement is that there be no isolated photon present in the barrel or end-cap shower counters. For this purpose, an isolated photon is defined by requiring that it have energy greater than $60 \mathrm{MeV}$; it must make an angle of greater than $12^{\circ}$ with respect to the original direction of each of the charged tracks, and also with respect to the direction defined for each charged track by connecting its point of entry to the barrel shower counter to the origin of the coordinate system. This reduces the data sample to $\sim 33000$ events. At this point there remains a significant number of cosmic ray events. The bulk of these are removed by rejecting any event for which either track has a measured time-of-flight value less than $2.5 \mathrm{~ns}$ or greater than 8.5 ns.

The particle identification procedure is applied to the $\sim 25000$ remaining events. For each allowed mass hypothesis $(e, \mu, \pi, K)$ for each track, the measured momentum is used to predict the expected values of $d E / d x$, time of flight, and shower counter energy. The corresponding measured quantities and resolutions are then used to create an overall $\chi^{2}$ value, which is converted to a confidence level using the number of contributing subdetectors as the number of degrees of freedom. The confidence level distributions found for samples of known pions, electrons, and muons within the momentum range accessible to $\tau$ decay are shown in Fig. 5. These distributions are all consistent with being flat, as would be expected for pure samples if the individual device resolutions were reliably assigned. Similar confidence level

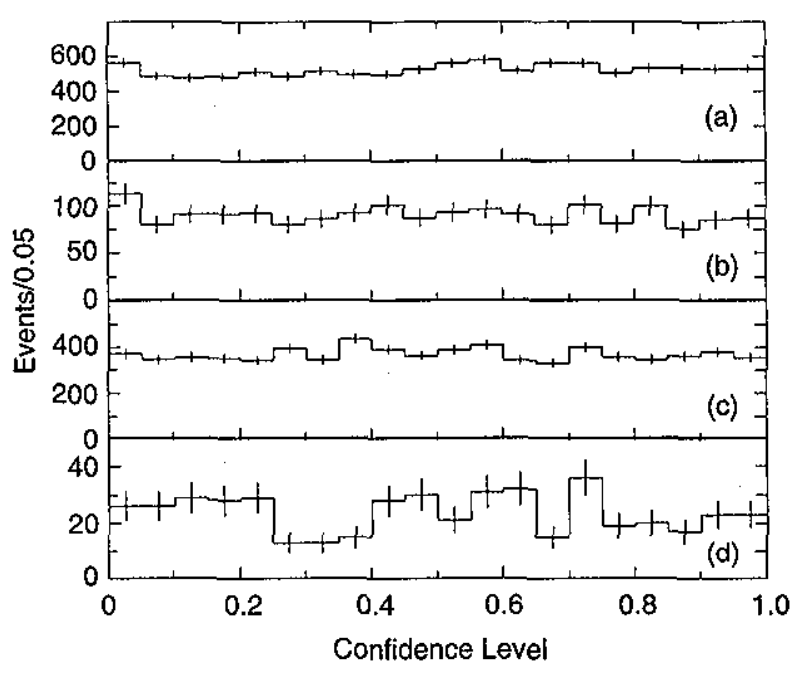

FIG. 5. The confidence level distributions for samples of (a) pions from $J / \psi \rightarrow \omega \pi \pi$ events and beam-gas electroproduction events, (b) electrons from radiative Bhabha events, (c) muons from $\mu$ pair production, and (d) muons from radiative $\mu$ pair production.

behavior is observed for each particle type in each individual detector system, confirming that this is indeed the case.

Events are rejected if either track has confidence levels of less than $5 \%$ for all particle hypotheses. Next, the $\mu$ hypothesis is assigned to a track if its momentum is greater than $500 \mathrm{MeV} / c$, it has confidence level greater than $5 \%$ as a $\mu$, and there are corroborating muon counter hits. Failing the $\mu$ requirement, a track is assigned either the $e$ or $h$ (for hadron, i.e., $\pi$ or $K$ ) hypothesis depending on which has the higher confidence level, and provided that confidence level is at least $5 \%$. If the confidence level for the $\pi(K)$ assignment is over $5 \%$, it is further required that the track momentum be consistent with two-body $\tau \rightarrow \pi \nu(K \nu)$ decay at the 3 $\sigma$ level. For the calculation of the relevant momentumu limits, $m_{\tau}$ is taken $1.0 \mathrm{MeV}$ below the previous measurement [3] to reduce the dependence of the $\tau \rightarrow \pi \nu(K \nu)$ selection efficiency on the c.m. energy; in extracting the $\tau$ mass value from the data, the small residual dependence is taken into account as described in Sec. VIII. The momentum limits for pions and kaons are shown as a function of c.m. energy in Fig. 6; for scan point 3, which is well below threshold [3], the threshold momentum limits are used. It should be emphasized that if, for a given track, either the $\pi$ or the $K$ hypothesis has confidence level greater than $5 \%$ and does not satisfy the momentum criterion, the event is rejected, even if the electron interpretation yields a higher confidence level.

More generally, for each event any mass hypothesis combination for which both tracks satisfy the $5 \%$ confidence level criterion is required to satisfy any subsequent selection criteria applied to that final state. If one such hypothesis fails this requirement, the event is rejected, 


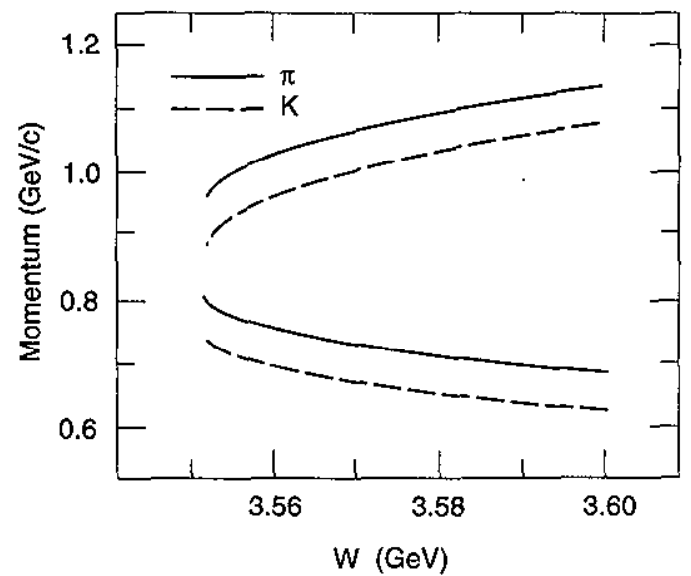

FIG. 6. The momentum limits as a function of c.m. energy $W$ for pions and kaons for the decays $\tau \rightarrow \pi \nu$ and $\tau \rightarrow K \nu$, respectively, obtained using a $\tau$ mass value of $1775.9 \mathrm{MeV}$, as discussed in the text.

even if another hypothesis is acceptable. This conservative approach is adopted since the main concern is to exclude background events from the $\tau^{+} \tau^{-}$data sample.

For example, the number of background events in the data sample is greatest at scan points 9 and 10 , for which the integrated luminosity is largest (see Table II). By design, these points are very close to the $\tau$ pair production threshold. If background events enter the $\tau^{+} \tau^{-}$sample at these points, they will drive the final mass to a value which is systematically low. It follows that the selection procedure followed in the analysis must be directed primarily toward the exclusion of background, while yielding an acceptable efficiency for the extraction of $\tau$ pair production events. The approach described in the previous paragraph is compatible with these goals.

Throughout the remainder of the analysis, each track is labeled by the most probable mass hypothesis, and the final state assigned accordingly. After the particle identification procedures; there remain $9080 \mathrm{ee}$ events, $73 \mu \mu$, events and 165 events in other final states.

\section{MONTE CARLO SIMULATION AND THE SELECTION OF THE FINAI $\tau^{+} \tau^{-}$EVENT SAMPLE}

In order to complete the $\tau^{+} \tau^{--}$event selection, it is necessary to compare the data sample obtained to this point in the analysis to an equivalent sample of Monte Carlo generated $\tau^{+} \tau^{-}$events. Consequently, the Monte Carlo program KORALB [13] is used to produce a sample of $100000 \tau$ pairs whose decays according to all known branching fractions [2] are fully simulated in the BES. Events are generated at each of the c.m. energies of the scan, and are distributed in number according to the product of luminosity and corrected cross section (i.e., the solid curve of Fig. 3) at each scan point; for this purpose, the threshold for $\tau$ pair production is chosen as $3553.8 \mathrm{MeV}$, as obtained in the previous $e \mu$ analy- sis [3]. The Monte Carlo events are reconstructed as for real data, and subjected to the selection criteria used to extract the $\tau^{+} \tau^{-}$candidate sample through the particle identification stage, as described in Sec. V.

The surviving Monte Carlo sample contains $17 \%$ ee events, $8 \% \mu \mu$ events, and $75 \%$ events in other final states. As indicated at the end of Sec V, the composition of the corresponding data sample is quite different, there being $\sim 5 / 9$ as many $\mu \mu$ events, and $\sim 70$ times as many $e e$ events, as events in other final states.

The $e e$ data sample results predominantly from twophoton $e^{+} e^{-} \rightarrow e^{+}\left(e^{-} e^{+}\right) e^{-}$events for which the leading $e^{+}$and $e^{-}$in the final state are undetected. The $\mu \mu$ sample similarly results primarily from $e^{+} e^{-} \rightarrow e^{+}\left(\mu^{+} \mu^{-}\right) e^{-}$ events, together with some few surviving cosmic rays. These background events are characterized by small net observed transverse momentum and, for the QED events, large missing energy. It follows that the variable PTEM, defined as

$$
\text { PTEM }=\frac{P_{x}}{E_{\text {miss }}^{\max }}=\frac{\left(\vec{P}_{1}+\vec{P}_{2}\right)_{x}}{W-\left|\overrightarrow{P_{1}}\right|-\left|\overrightarrow{P_{2}}\right|}
$$

(i.e., the ratio of the net observed transverse momentum to the maximum possible value of the missing energy), is localized to small values for QED background events; this is shown explicitly in Fig. 7(a) for the $\tau^{+} \tau^{-}$candidate events. The corresponding distribution for the $\tau^{+} \tau^{-}$Monte Carlo sample is shown in Fig. 7(b); the contrast between these distributions is dramatic, and it is clear that the purity of the $\tau^{+} \tau^{-}$data sample can be greatly enhanced by removing candidates at small values of PTEM.

Since the ee final state in the data sample appears to consist almost entirely of background, it is considered first. This is necessary, not because of the resulting direct contribution of $e e$ events to the $\tau$ mass measurement (which proves to be small), but in order to prevent ee background events from feeding through to other final states as a consequence of particle misidentification.

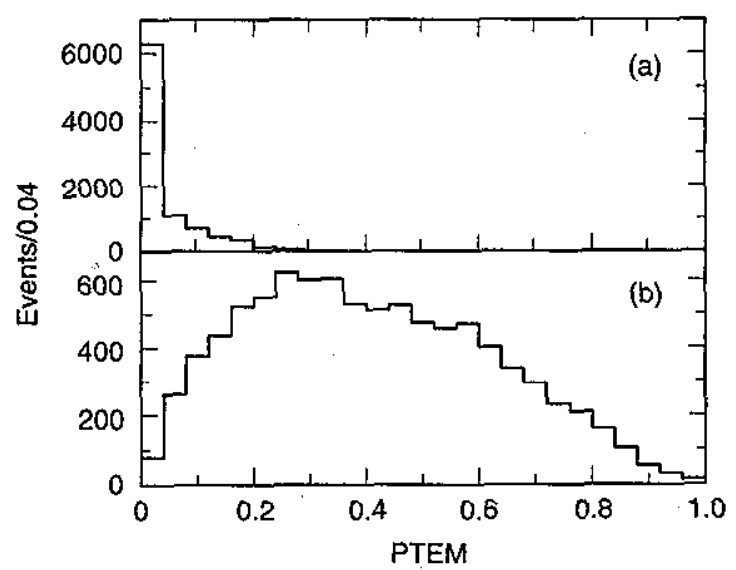

FIG. 7. The distribution in the variable PTEM for (a) the $\tau^{+} \tau^{-}$candidate sample after the application of the particle identification criteria and (b) $\tau^{+} \tau^{-}$events from the Monte Carlo simulation selected by the same criteria. 
For this purpose, it proves useful to examine the correlation between PTEM and the acoplanarity angle $\theta_{\text {acop }}$ [14]. This is shown for $e e$ candidate events, and for $e e$ events from the $\tau^{+} \tau^{-}$Monte Carlo sample in Figs. 8(a) and $8(b)$, respectively. The distributions are very different, confirming that the $e e$ data sample consists mainly of background.

The events of Fig. 8(a) have been subjected to an additional selection procedure beyond particle identification. For an event at an intermediate value of PTEM $(\geq 0.2)$, an enlarged version of the event display in $x-y$ projection sometimes reveals the presence of a low-angle, third charged track which is impossible to reconstruct fully. Such a track is characterized by the presence of radially aligned hits in the four layers of the CDC, followed by hits in the first two MDC layers; frequently, related hits in the end-cap TOF and shower counters provide further corroboration. An example is shown in Fig. 9.

Clearly, such three-prong events should be rejected from the $\tau^{+} \tau^{-}$data sample. Consequently, for each $e e$ candidate with PTEM $\geq 0.2$, an enlarged view of the event is examined prior to preparing Fig. 8(a); 100 events showing evidence for a low-angle, third charged track are rejected, and this serves to better define the separation between the signal and background regions. Events having PTEM $<0.2$ are not checked because the level of background makes it impossible to isolate a clean $\tau^{+} \tau^{-}$ sample in this region.

In Fig. 8(a), it is clear that background continues to dominate for PTEM values greater than 0.2 and acoplanarity $\sim 180^{\circ}$. Since the primary concern is to exclude background events from the $\tau^{+} \tau^{-}$sample, only those $e e$ events having PTEM $\geq 0.3$ and acoplanarity angle $\leq 160^{\circ}$ are retained; $6 e e$ events survive, and the candi-

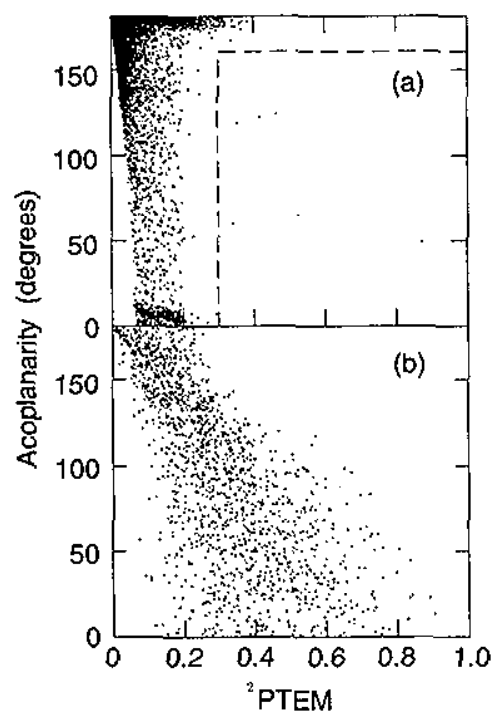

FIG. 8. (a) The scatterplot of PTEM vs acoplanarity for the $\tau^{+} \tau^{-}$candidate ee events; the dashed lines denote the region $\mathrm{PTEM} \geq 0.3$ and acoplanarity $\leq 160^{\circ}$, within which events are retained. (b) The same scatterplot for ee events obtained from the $\tau^{+} \tau^{-}$Monte Carlo simulation after the application of the same selection criteria.

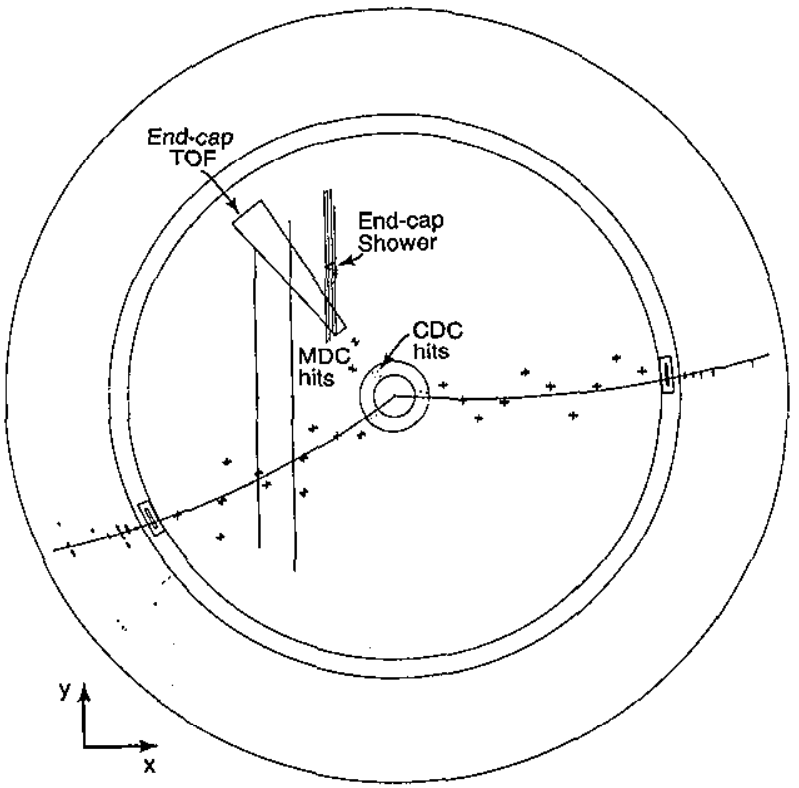

FIG. 9. A $\tau^{+} \tau^{-}$candidate event in $x-y$ projection, with evidence for a low-angle, third charged track, as indicated by the four hits in the CDC, followed by hits in layers 1 and 2 of the MDC; the associated hits in the end-cap TOF and shower counters further corroborate the presence of a third charged track.

date event sample is reduced to 244 events as a result. Furthermore, since the high density of $e e$ events in the excluded region of Fig. 8(a) might yield background contributions to other final states through particle misidentification, any event in the excluded region having electron confidence level $\geq 5 \%$ for both tracks is also eliminated, even if the ee interpretation is not the most probable one. This conforms to the discussion at the end of Sec. V, and reduces the data sample to 199 events.

The surviving nonee events exhibiting a low-angle, third charged track, as described for the ee candidates, are rejected. At the same time, the few surviving cosmic ray events are removed from the $\mu \mu$ sample; these are readily recognized by their back-to-back track configuration, the absence of TOF information on both tracks, and, frequently, the presence of nonradial CDC hits connecting the two tracks.

The PTEM vs acoplanarity scatterplots for the 140 remaining $\mu \mu, e \mu$, and combined $e h, \mu h$, and $h h$ candidates are shown in Figs. 10(a), 11(a), and 12(a), respectively; the corresponding Monte Carlo distributions are shown in Figs. 10(b), 11(b), and 12(b). It should be pointed out that the Monte Carlo events in these figures and in Fig. $8(\mathrm{~b})$ are not examined individually for the presence of a low-angle, third charged track. However, the effect of such a procedure is simulated by rejecting events reconstructed as two prongs for which at least one other track generates hits in the CDC and in the first two layers of the MDC. Only $1.6 \%$ of the reconstructed Monte Carlo two-prong events are discarded for this reason.

On the basis of the comparison of the data and Monte 


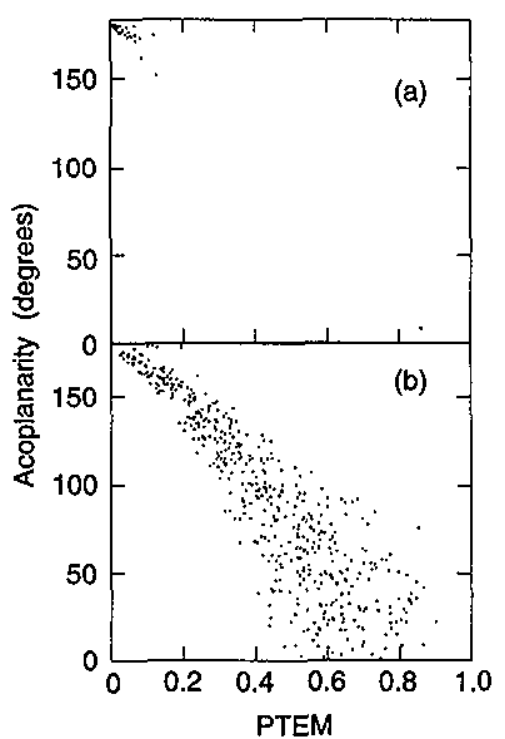

FIG. 10. (a) The scatterplot of PTEM vs acoplanarity for the $\tau^{+} \tau^{-}$candidate $\mu \mu$ events. (b) The same scatterplot for $\mu \mu$ events obtained from the $\tau^{+} \tau^{-}$Monte Carlo simulation after the application of the same selection criteria.

Carlo scatterplots, only those $\mu \mu$ events for which PTEM $\geq 0.2$ are retained [Fig. 10(a)]. The $e \mu$ sample of Fig. 11(a) seems free of background, and so no PTEM criterion is imposed, while for the data of Fig. 12(a), the events for which PTEM $<0.1$ are excluded from the final sample. After removal of the events failing to satisfy the PTEM criteria, there remain only 65 events in the $\tau^{+} \tau^{-}$ candidate sample.

The effect of the sequential event selection criteria is

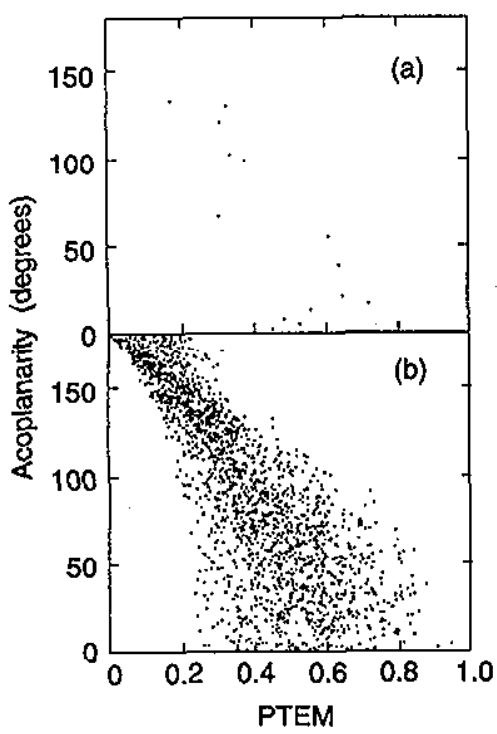

FIG. 11. (a) The scatterplot of PTEM vs acoplanarity for the $\tau^{+} \tau^{-}$candidate $e \mu$ events. (b) The same scatterplot for $e \mu$ events obtained from the $\tau^{+} \tau^{-}$Monte Carlo simulation after the application of the same selection criteria.

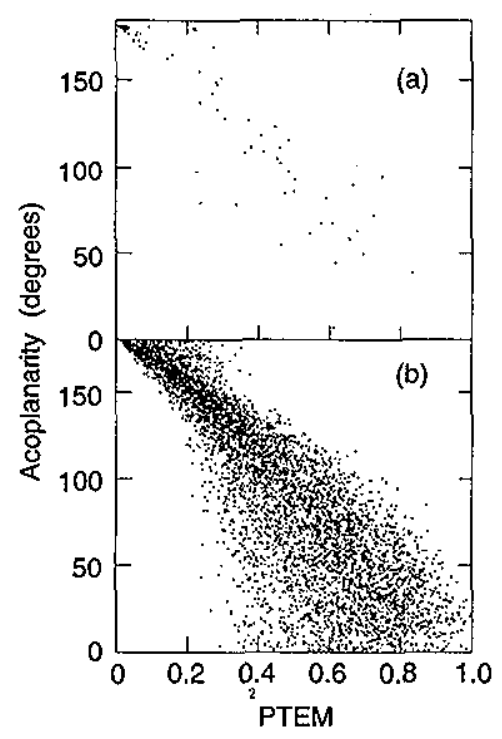

FIG. 12. (a) The scatterplot of PTEM vs acoplanarity for the $\tau^{+} \tau^{-}$candidate $e h, \mu h$, and $h h$ events. (b) The same scatterplot for the combined $e h, \mu h$, and $h h$ events obtained from the $\tau^{+} \tau^{-}$Monte Carlo simulation after the application of the same selection criteria.

summarized in Table III, and the event display for a typical surviving $e \mu$ event is shown in x-y projection in Fig. 13. The events of Figs. 8(a), 10(a), 11(a), and 12(a) are distributed by final state as indicated in the second

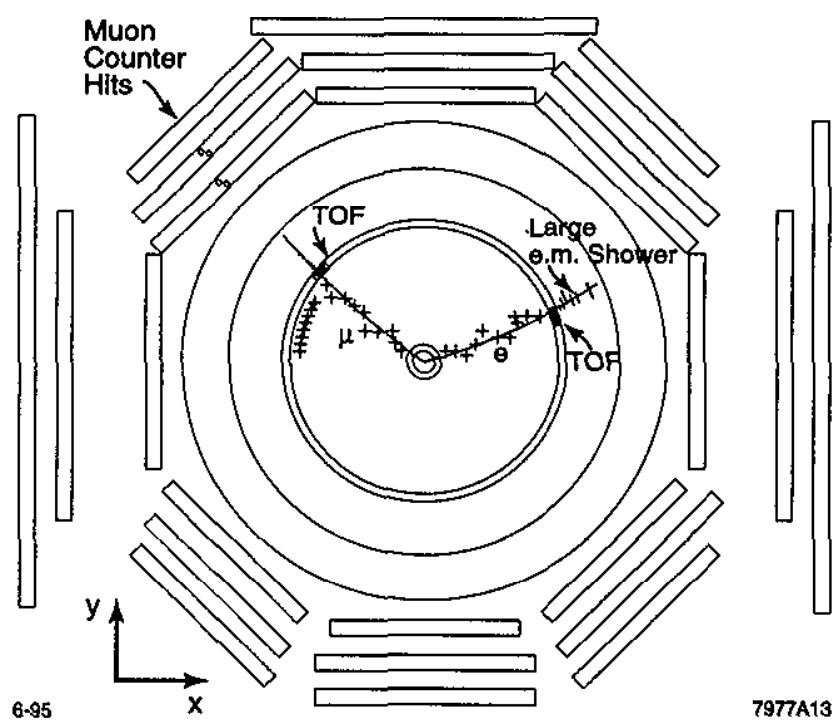

FIG. 13. A typical $e \mu$ candidate event in $x-y$ projection. The MDC hits provide $d E / d x$ information on each track; in addition, both tracks activate the barrel TOF counters as indicated, and hence have associated velocity measurements. The electron track ends in a large shower in the electromagnetic calorimeter, which contributes significantly to its identification as an electron, while the muon track penetrates the shower counter, the magnet coil, and two layers of the muon system. 
TABLE III. The effect of the sequential event selection criteria on the data sample (initially, $11.7 \times 10^{6}$ events).

\begin{tabular}{|c|c|}
\hline Selection criteria & Events remaining \\
\hline $\begin{array}{l}\text { Two charged tracks with vertex in the interaction region } \\
|\cos \theta|<0.75 \text { for each track }\end{array}$ & 470000 \\
\hline$P_{T} \geq 100 \mathrm{MeV} / c ;|\vec{P}| \leq P_{\max }\left(W, m_{\tau}\right)+3 \sigma_{P}$ & 40000 \\
\hline $\begin{array}{l}\text { Removal of events with } \geq 1 \text { isolated photon } \\
{\left[E_{\gamma} \geq 60 \mathrm{MeV} \text { and } \min \left\{\cos ^{-1}\left(\widehat{P_{\gamma}} \cdot \widehat{P}\right)\right\} \geq 12^{\circ}\right]}\end{array}$ & 33000 \\
\hline Removal of cosmic ray events (TOF $\leq 2.5 \mathrm{~ns}$ or $\mathrm{TOF} \geq 8.5 \mathrm{~ns}$ ) & 25000 \\
\hline Final state particle ID; hadron momentum requirement for $\tau \rightarrow h \nu$ & 9318 \\
\hline $\begin{array}{l}\text { Removal of ee events with PTEM } \geq 0.2 \text { and a } \\
\text { low-angle, third charged track }\end{array}$ & 9218 \\
\hline Removal of ee events with $\mathrm{PTEM}<0.3$ or $\theta_{\text {acop }}>160^{\circ} \quad(6$ ee events survive $)$ & 244 \\
\hline $\begin{array}{l}\text { Removal of any non-ee event with an acceptable } e e \text { interpretation } \\
\text { having PTEM }<0.3 \text { or } \theta_{\text {acop }}>160^{\circ}\end{array}$ & 199 \\
\hline $\begin{array}{l}\text { Removal of Non-ee events with low-angle, third charged track } \\
\text { removal of residual cosmic ray } \mu \mu \text { events }\end{array}$ & 146 \\
\hline Non-ee PTEM requirements & 65 \\
\hline
\end{tabular}

column of Table IV. The relevant PTEM criteria are listed in the third column, and the number of candidates surviving is shown in column 4 ; these selected events are distributed by final state and scan point as indicated in Table V.

None of the surviving candidates has net charge \pm 2 , even though the requirement of charge balance is not imposed. This shows explicitly that the $\tau^{+} \tau^{-}$selection criteria successfully remove background contributions from two-photon events of the type $e^{+} e^{-} \rightarrow e^{+} e^{-} X^{+} X^{-}$to the $e X$ final states, since same-sign and opposite-sign contributions from such a source are equally likely to occur.

For the Monte Carlo sample, 7588 events survive the selection criteria. Only two events have net charge \pm 2 , and these were generated as four-prong events (i.e., one $r$ decayed to the one-prong topology, and the other to the three-prong topology). The remaining events are generated and reconstructed as two-prong events with zero net charge.

\section{COMPARISON OF THE DATA AND EQUIVALENT MONTE CARLO SAMPLES}

The PTEM vs acoplanarity scatterplot for the final $\tau^{+} \tau^{-}$data sample is shown in Fig. 14(a) in comparison to that for the Monte Carlo sample [Fig. 14(b)] after application of the same selection criteria. There is very good qualitative agreement.

The distributions in acoplanarity [14], PTEM, and track momentum for the $\tau^{+} \tau^{-}$candidate sample (points) are compared to the corresponding Monte Carlo distribu-

TABLE IV. The number of $\tau^{+} \tau^{-}$candidate events by final state before and after PTEM selection.

\begin{tabular}{cccc}
\hline $\begin{array}{l}\text { Final } \\
\text { state }\end{array}$ & $\begin{array}{c}\text { Number of events } \\
\text { before PTEM selection }\end{array}$ & $\begin{array}{c}\text { Minimum value } \\
\text { of PTEM }\end{array}$ & $\begin{array}{c}\text { Events remaining } \\
\text { after PTEM selection }\end{array}$ \\
\hline$e e$ & 8980 & 0.3 and $\theta_{\text {acop }} \leq 160^{\circ}$ & 6 \\
$e \mu$ & 14 & 0.0 & 14 \\
$e h$ & 25 & 0.1 & 21 \\
$\mu \mu$ & 51 & 0.2 & 2 \\
$\mu h$ & 26 & 0.1 & 11 \\
$h h$ & 24 & 0.1 & 11 \\
\hline \hline
\end{tabular}


TABLE $\mathrm{V}$. The number of $\tau^{+} \tau^{-}$candidate events by final state and scan point after selecting on PTEM at the values listed in Table IV.

\begin{tabular}{cccccccc}
\hline \hline Scan point & All final states & $e e$ & $e \mu$ & $e h$ & $\mu \mu$ & $\mu h$ & $h h$ \\
\hline 1 & 9 & & 2 & 3 & & 2 & 2 \\
2 & 7 & & 2 & 3 & 1 & 1 & \\
3 & 0 & & & & & & \\
4 & 0 & & & & & & \\
5 & 5 & & 2 & 2 & & 1 & \\
6 & 1 & 1 & & & & & \\
7 & 2 & 1 & & & & 1 & \\
8 & 1 & & 1 & & & & \\
9 & 1 & & & 1 & & & \\
10 & 3 & & 1 & 1 & & & 1 \\
11 & 24 & 2 & 5 & 8 & 1 & 2 & 6 \\
12 & 12 & 2 & 1 & 3 & & 4 & 2 \\
\hline Total & 65 & 6 & 14 & 21 & 2 & 11 & 11 \\
\hline \hline
\end{tabular}

tions (histograms) normalized to the data in Figs. 15-17. The quantitative agreement is very good in all cases.

The data distribution by final state is compared to that for the Monte Carlo sample of Fig. 14(b) (normalized to 65 events) in Table VI. Again there is very good quantitative agreement.

The final state assignments of Tables IV-VI and Figs. 8 and 10-12 are not to be taken literally for either data or Monte Carlo events. This is shown explicitly for the Monte Carlo data in Table VII, which indicates the correlation between the generated and assigned final states, with the total normalized to the 65 events in the final $\tau^{+} \tau^{-}$data sample. The main areas of misidentification involve the $e \mu$ and $e h, \mu \mu$ and $\mu h$, and $\mu h$ and $h h$ final states.

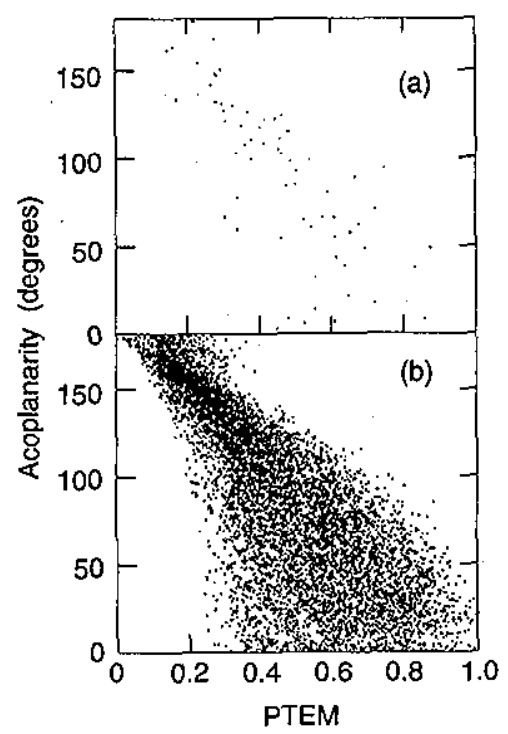

FIG. 14. (a) The scatterplot of PTEM vs acoplanarity for the final $\tau^{+} \tau^{-}$candidate event sample. (b) The same scatterplot for events obtained from the $\tau^{+} \tau^{-}$Monte Carlo simulation after the application of the same selection criteria.
TABLE VI. The comparison to the event distribution by final state to that from the accepted $\tau^{+} \tau^{-}$Monte Carlo sample, normalized to 65 events.

\begin{tabular}{ccc}
\hline \hline Final state & Data & Monte Carlo \\
\hline$e e$ & 6 & 5.4 \\
$e \mu$ & 14 & 15.9 \\
$e h$ & 21 & 19.1 \\
$\mu \mu$ & 2 & 3.8 \\
$\mu h$ & 11 & 12.4 \\
$h h$ & 11 & 8.4 \\
\hline
\end{tabular}

A muon can be misidentified as a hadron since the solid angle coverage lof the muon counter system is sinaller than that within which tracks can be reconstructed; i.e., true muon tracks can be found which miss the muon counters. Since the other particle systems do not distinguish a $\mu$ from a $\pi$, and since $\mu$ identification requires corroborating muon hits, such a track is usually misidentified as a $\pi$, and sometimes as a $K$.

Conversely, a $\pi$ or $K$ can be misidentified as a $\mu$ by penetrating the material prior to the muon counter system with sufficient residual energy to generate hits in the muon detector. By using identified pions, the rate at which this occurs is found to vary with momentuxn from $\sim 7 \%$ at $0.6 \mathrm{GeV} / c$ to $\sim 15 \%$ at $1.0 \mathrm{GeV} / c$. This, together with the momentum distribution of Fig. 17, implies an overall $\mu-h$ misidentification rate of $\sim 10 \%$, in agreement with the data of Table VII.

Since the migration matrix (Table VII) is approximately diagonal, the net effect of misidentification on the event rates to the individual final states is quite small. If follows that, although individual events in the final data sample may be assigned to the incorrect final state, the measured relative rates in Table VI are reliable and, within the associated Poisson uncertainties, are consistent with the underlying $\tau$ branching fraction data [2] embodied in the Monte Carlo generator.

This is not the case, however, within the category of tracks classified as hadrons. Of the Monte Carlo tracks generated as pions, $65 \%$ are correctly identified by the selection program, and these represent $86 \%$ of all tracks assigned the pion identity. For kaons, $76 \%$ of the generated kaons are correctly identified; however, these con-

TABLE VII. The correlation between the generated and assigned final states for the accepted $\tau^{+} \tau^{-}$Monte Carlo sample, normalized to 65 events.

\begin{tabular}{|c|c|c|c|c|c|c|}
\hline \multirow[b]{2}{*}{ Assigned } & \multicolumn{6}{|c|}{ Generated } \\
\hline & $e e$ & $e \mu$ & $e h$ & $\mu \mu$ & $\mu h$ & $h h$ \\
\hline$e e$ & 5.24 & 0.04 & 0.09 & 0.01 & 0.00 & 0.00 \\
\hline$e \mu$ & 0.00 & 13.90 & 1.85 & 0.08 & 0.06 & 0.01 \\
\hline$e h$ & 0.08 & 2.03 & 16.76 & 0.01 & 0.09 & 0.09 \\
\hline$\mu \mu$ & 0.00 & 0.00 & 0.00 & 2.99 & 0.70 & 0.06 \\
\hline$\mu h$ & 0.00 & 0.04 & 0.00 & 1.29 & 10.09 & 1.03 \\
\hline$h h$ & 0.00 & 0.01 & 0.11 & 0.15 & 1.68 & 6.40 \\
\hline Total generated & 5.32 & 16.02 & 18.81 & 4.52 & 12.62 & 7.59 \\
\hline
\end{tabular}




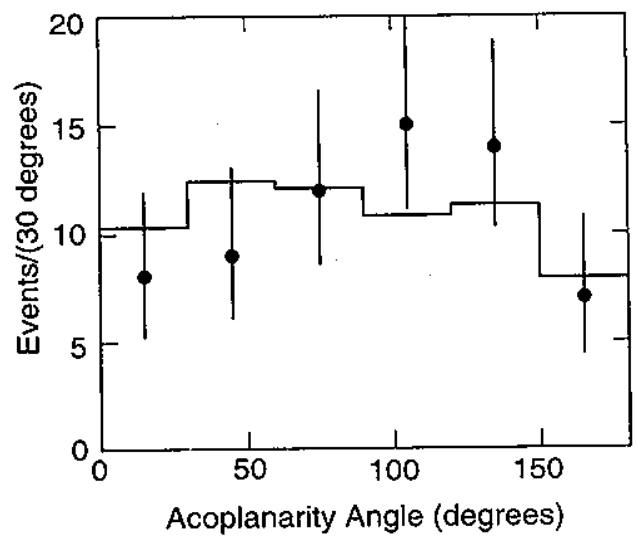

FIG. 15. The distribution in acoplanarity angle for the $\tau^{+} \tau^{-}$candidate events (points) compared to that for the $\tau^{+} \tau^{-}$Monte Carlo events subjected to the same selection criteria and normalized to the data (histogram).

stitute only $11 \%$ of all tracks assigned as kaons, $80 \%$ of which are actually misidentified pions. In contrast, $88 \%$ of the tracks generated as hadrons are actually assigned to this category.

The excellent agreement between data and Monte Carlo samples evident in Figs. 15-17 and in Table VI indicates that the pion and kaon identification problems do not adversely affect the ability of the selection criteria to extract $\tau^{+} \tau^{-}$events from the data. Consequently, this sample is used as described in Sec. VIII to obtain a measurement of the mass of the $\tau$ lepton.

\section{MAXIMUM LIKELIHOOD FIT TO THE DATA}

The mass of the $\tau$ lepton is obtained from the final $\tau^{+} \tau^{-}$candidate sample by means of a maximum likelihood fit to the c.m. energy dependence of the $\tau$ pair

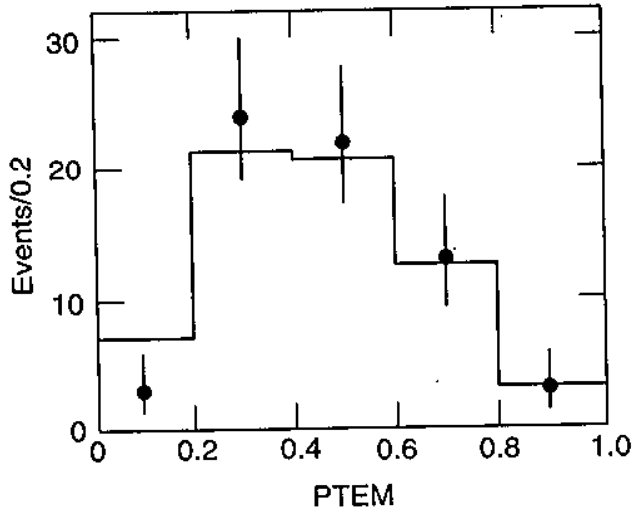

FIG. 16. The distribution in PTEM for the $\tau^{+} \tau^{-}$candidate events (points) compared to that for the $\tau^{+} \tau^{-}$Monte Carlo events subjected to the same selection criteria and normalized to the data (histogram).

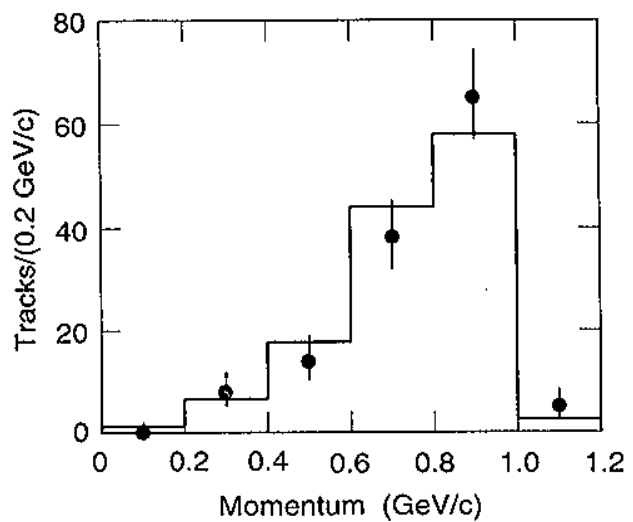

FIG. 17. The distribution in momentum for the tracks from the $\tau^{+} \tau^{-}$candidate events (points) compared to that for the $\tau^{+} \tau^{-}$Monte Carlo events subjected to the same selection criteria and normalized to the data (histogram).

production cross section. The likelihood function is a product of Poisson distributions, one at each of the 12 scan points, and takes the form

$$
L\left(m_{\tau}, \epsilon, \sigma_{B}\right)=\prod_{i=1}^{12} \frac{\mu_{i}^{N_{i}} e^{-\mu_{i}}}{N_{i} !},
$$

where $N_{i}$ is the number of observed $\tau^{+} \tau^{-}$events at scan point $i ; \mu_{i}$ is the corresponding number of events expected, and is given by

$$
\mu_{i}=\left[\varepsilon r_{i} \sigma\left(W_{i}, m_{\tau}\right)+\sigma_{B}\right] \mathcal{L}_{i} .
$$

In Eq. (11), $m_{\tau}$ is the mass of the $\tau$ lepton, and $\epsilon$ is the overall efficiency for identifying $\tau^{+} \tau^{-}$events; this includes branching fractions, and allows for uncertainties in luminosity scale, and in trigger and detector efficiency; the $r_{i}$ are the point-to-point relative efficiency values measured using the Monte Carlo data surviving the $\tau$ event selection criteria; for scan points $1,2,5,11$, and 12, $r_{i} \sim 1.0$, while for the remaining points in the immediate vicinity of threshold, $r_{i} \sim 0.9 ; \sigma_{B}$ is an effective background cross section, and is assumed constant over the limited range of c.m. energy, $W_{i}$, covered by the scan; $\mathcal{L}_{i}$ is the integrated luminosity at scan point $i$, and $\sigma\left(W_{i}, m_{\tau}\right)$ is the corresponding cross section for $\tau^{+} \tau^{-}$production corrected for Coulomb interaction, initial and final state radiation, vacuum polarization, and beam energy spread, as given by Eqs. (7) and (8), and represented by the solid curve of Fig. 3 .

As a test of the procedure, the likelihood fit is performed on the selected Monte Carlo data sample. With the background cross section set to zero, the value of $m_{\tau}$ used in generating the events is recovered to within 0.005 $\mathrm{MeV}$. If the relative efficiency factors $r_{i}$ in Eq. (11.) are set to 1.0 , the fitted value of $m_{\tau}$ is found to be $0.06 \mathrm{MeV}$ higher than the input value; this demonstrates the need to take account of the point-to-point relative efficiency.

In carrying out the maximum likelihood fit, $m_{\tau}, \epsilon$, and 
$\sigma_{B}$ are allowed to vary, subject to the requirement $\sigma_{B} \geq$ 0 . The fit to the data of Table $\mathrm{V}$ is performed using the program MINUIT [15], and the maximum likelihood solution is found to correspond to the parameter values

$$
\begin{aligned}
m_{\tau} & =1776.96_{-0.21}^{+0.18} \mathrm{MeV}, \\
\epsilon & =4.26_{-0.51}^{+0.55} \%, \\
\sigma_{B} & =0^{+0.74} \mathrm{pb} .
\end{aligned}
$$

For $\epsilon$ and $\sigma_{B}$, the quoted uncertainties are obtained by fixing the other two parameters at their maximum likelihood values and finding the parameter values corresponding to a decrease in $\ln L$ of 0.5 .

The statistical uncertainty in $m_{\tau}$ is found by setting $\epsilon=4.26 \%, \sigma_{B}=0$, and integrating the likelihood function to find the $68.27 \%$ confidence level interval; i.e., for $m<m_{\tau}$, the $1 \sigma$ exror point, $m_{\text {low }}$, is defined by

$$
\int_{m_{\text {low }}}^{m_{\tau}} L d m=0.6827 \int_{0}^{m_{\tau}} L d m
$$

and for $m>m_{\tau}$, the $1 \sigma$ error point $m_{\text {high }}$ is defined by

$$
\int_{m_{\tau}}^{m_{\mathrm{high}}} L d m=0.6827 \int_{m_{\tau}}^{\infty} L d m .
$$

For a Gaussian likelihood function, these error estimates would be the same as those obtained from a decrease in $\ln L$ of 0.5 ; the procedure embodied in Eqs. (13) and (14) attempts to take account of any non-Gaussian behavior. The likelihood function in the present analysis actually exhibits a mass dependence which is close to Gaussian [cf. Fig. 18(c)]; a decrease in $\ln L$ of 0.5 yields error estimates of ${ }_{-0.201}^{+0.195} \mathrm{MeV}$, so that in this instance there is very little difference in the results of the two methods.

The quality of the fit is checked by forming the likelihood ratio $\lambda$ with the result $-2 \ln \lambda=2.1$; in the large statistics limit, this should obey a $\chi^{2}$ distribution for nine degrees of freedom, which implies that a very good fit has been achieved. This is shown explicitly in Figs. 18(a) and 18(b). The curve corresponds to the cross section given by Eqs. (7) and (8) with $m_{\tau}=1776.96 \mathrm{MeV}$; the measured value at scan point $i$ is given by

$$
\sigma_{i}=\frac{N_{i}}{\epsilon r_{i} \mathcal{L}}
$$

since $\sigma_{B}=0$ from the fit, and the error bars result primarily from the Poisson errors on $N_{i}$; these are obtained excluding the value $N_{i}$ from the $68.27 \%$ confidence interval of the Poisson probability distribution.

In Fig. 18(c), the dependence of $\ln L$ on $m_{\tau}$ in the present analysis is compared to that obtained in the previous $e \mu$ analysis [3]. The maxima are clearly consistent, but the present analysis yields a much narrower and more symmetric distribution as a consequence of the much larger data sample obtained.
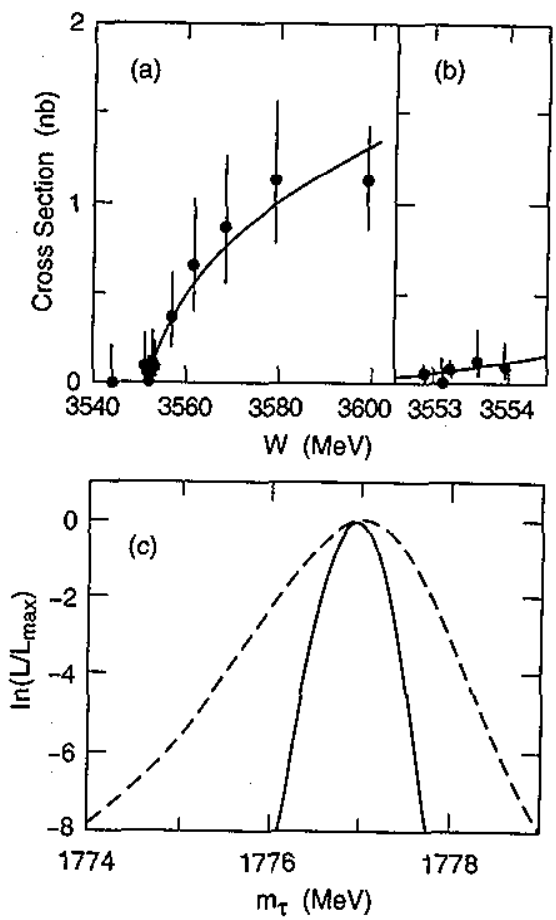

FIG. 18. (a) The c.m. energy dependence of the $\tau^{+} \tau^{-}$ cross section resulting from the likelihood fit (curve), compared to the data (Poisson errors). (b) An expanded version of (a), in the immediate vicinity of $\tau^{+} \tau^{-}$threshold. (c) The solid curve shows the dependence of the logarithm of the likelihood function on $m_{r}$, with the efficiency and background parameters fixed at their most likely values; the dashed curve shows the likelihood function from Ref. [3].

\section{SOURCES OF SYSTEMATIC UNCERTAINTY}

Five sources of systematic uncertainty are considered: the fitted efficiency parameter $\epsilon$, which, by definition, incorporates the uncertainties in luminosity scale, and also in trigger and detection efficiency (see Sec. VIII); the effective background cross section $\sigma_{B}$; possible bias in the c.m. energy scanning procedure; the c.m. energy scale; and the spread in c.m. energy.

The systematic uncertainties associated with the fitted efficiency parameter are obtained by setting $\epsilon$ at its $\pm 1 \sigma$ values and maximizing the likelihood with respect to $m_{r}$ with $\sigma_{B}=0$. This yields changes in the fitted $\tau$ mass of $\Delta m_{\tau}={ }_{-0.10}^{+0.09} \mathrm{MeV}$.

In a similar way, the uncertainty in $m_{\tau}$ associated with $\delta \sigma_{B}=+0.74 \mathrm{pb}$ (corresponding to a $1 \sigma$ background level of 3.8 events) is found by setting $\sigma_{B}$ to this value, fixing $\epsilon=4.26 \%$, and maximizing the likelihood with respect to $m_{\tau}$; this yields a systematic error contribution $\Delta m_{\tau}=$ $+0.19 \mathrm{MeV}$.

The systematic uncertainty associated with possible bias in the scanning procedure has been studied [16]. A large number of Monte Carlo simulations of the stepping procedure used to locate the $\tau$ pair production threshold has been carried out under varying initial conditions, and corresponding $\tau$ mass values extracted. The conclu- 
TABLE VIII. The measured mass and c.m. energy spread determined from fits to the hadronic cross section observed in the $J / \psi$ resonance scans performed during data acquistion.

\begin{tabular}{lr}
\hline \hline Fitted $J / \psi$ mass $(\mathrm{MeV})$ & c.m. energy spread $(\mathrm{MeV})$ \\
\hline $3097.32 \pm 0.02$ & $1.04 \pm 0.017$ \\
$3097.07 \pm 0.02$ & $1.20 \pm 0.016$ \\
\hline
\end{tabular}

sion is that the degree of bias in the mass value obtained in this way is very small, and contributes a systematic uncertainty of $\pm 0.10 \mathrm{MeV}$ to the measurement.

Finally, the systematic errors due to uncertainties in the c.m. energy scale and energy spread are discussed. The energy scale is determined from several scans of the $J / \psi$ and $\psi^{\prime}$ resonances performed during the experiment [see Fig. 4 (a)]. The plots of observed hadron cross section vs c.m. energy for two of the five scans are shown in Fig. 19. From the data in Table VIII the values $M_{\psi}=3097.20 \mathrm{MeV}$ and $\delta M_{\psi}=$ $|3097.32-3097.07| / \sqrt{2}=0.18 \mathrm{MeV}$ are obtained, and from the data in Table IX, $M_{\psi^{\prime}}=3686.88 \mathrm{MeV}$ and $\delta M_{\psi^{\prime}}=\sqrt{(0.15)^{2}+0.0^{2}+(0.14)^{2}} / \sqrt{2}=0.15 \mathrm{MeV}$. The uncertainties in the energy scale, including the uncertainty in the reproducibility of the BEPC energy settings listed above, are listed in Table X. Assuming a linear relation between measured energy $W_{M}$ and the corrected value $W$, the latter is given by

$$
W=T_{\psi}+\left(W_{M}-M_{\psi}\right)\left(\frac{T_{\psi^{\prime}}-T_{\psi}}{M_{\psi^{\prime}}-M_{\psi}}\right)
$$

The resulting c.m. energy scale correction at $\tau^{+} \tau^{-}$ threshold is $W-W_{M}=-0.754 \mathrm{MeV}$, with corresponding uncertainty $\delta W=0.173 \mathrm{MeV}$. This is included as a systematic uncertainty of $\Delta m_{\tau}=\delta W / 2= \pm 0.09 \mathrm{MeV}$.

Fits to the two resonances are used to measure the beam energy spread, and its variation with c.m. energy and beam current [12]. The resulting uncertainty in c.m. energy spread is estimated to be $\pm 0.08 \mathrm{MeV}$. By varying the spread parameter over this range, and repeating the likelihood fits, the corresponding uncertainty in the $\tau$ mass value is found to be $\Delta m_{r}= \pm 0.02 \mathrm{MeV}$.

The independent systematic error contributions are summarized in Table XI. Added in quadrature, they yield a total systematic mass uncertainty $\Delta m_{\tau}=$ ${ }_{-0.17}^{+0.25} \mathrm{MeV}$. It follows from Eq. (12) that, as a result of the improved statistics in the present analysis, the statistical and systematic uncertainties associated with the tau mass value are now comparable; the consequence is

TABLE IX. The measured mass and c.m. energy spread determined from fits to the hadronic cross section observed in the $\psi^{\prime}$ resonance scans performed during data acquistion.

\begin{tabular}{lr}
\hline \hline Fitted $\psi^{\prime}$ mass $(\mathrm{MeV})$ & c.m. energy spread $(\mathrm{MeV})$ \\
\hline $3687.03 \pm 0.03$ & $1.49 \pm 0.043$ \\
$3686.88 \pm 0.03$ & $1.34 \pm 0.038$ \\
$3686.74 \pm 0.03$ & $1.40 \pm 0.024$ \\
\hline
\end{tabular}

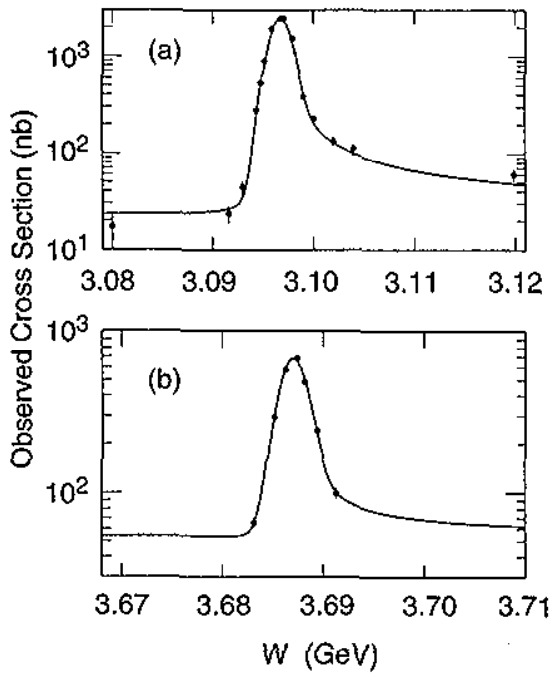

FIG. 19. The results of typical scans, taken during the experiment of (a) the $J / \psi$ resonance and (b) the $\psi^{\prime}$ resonance; $W$ is the c.m. energy.

an improvement in overall precision by a factor of 2 with respect to the previous $\mathrm{BES}$ measurement [3].

\section{CONCLUSION}

By means of a maximum likelihood fit to $\tau^{+} \tau^{-}$cross section data near threshold, the mass of the $\tau$ lepton

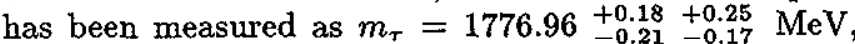
where the first uncertainties are statistical and the second systematic. This measurement is shown with all other measurements of the mass of the $\tau$ lepton [2] in Fig. 20. Inserting this new value into Eq. (6), together with the values $t_{\tau}=(291.6 \pm 1.7)$ fs and $B_{\tau \rightarrow e \nu \bar{\nu}}=(17.66 \pm 0.11) \%$ [17], the ratio of squared coupling constants becomes

$$
\left(G_{\tau \rightarrow e \nu \nu} / G_{\mu \rightarrow e \nu \nu}\right)^{2}=0.9886 \pm 0.0085,
$$

so that this test of $\tau \mu$ universality is satisfied at the 1.3 standard deviation level.

The consistency of $m_{\tau}, t_{\tau}$, and $B_{\tau \rightarrow e \nu \nu}$ with $\tau \mu$ universality is shown graphically in Fig. 21. From Eq. (6), the values of $t_{\tau}$ and $B_{\tau \rightarrow e \nu \bar{\nu}}$ should lie on a line with slope proportional to $m_{\tau}^{-5}$ through the origin. The solid lines in Fig. 21 indicate the $\pm 1 \sigma$ band obtained by using the measurement of $m_{\tau}$ from the present analysis and by

TABLE X. The contributions to the uncertainty in the c.m. energy scale.

\begin{tabular}{llc}
\hline \hline Quantity & Error (MeV) \\
\hline$W_{M}$ & $:$ BEPC measured c.m. energy & 0.10 \\
$M_{\psi}$ & $:$ BES value for $J / \psi$ mass & 0.18 \\
$M_{\psi^{\prime}}$ & $:$ BES value for $\psi^{\prime}$ mass & 0.15 \\
$T_{\psi}$ & $:$ Nominal value for $J / \psi$ mass $[2]$ & 0.04 \\
$T_{\psi^{\prime}}$ & $:$ Nominal value for $\psi^{\prime}$ mass [2] & 0.09 \\
\hline \hline
\end{tabular}




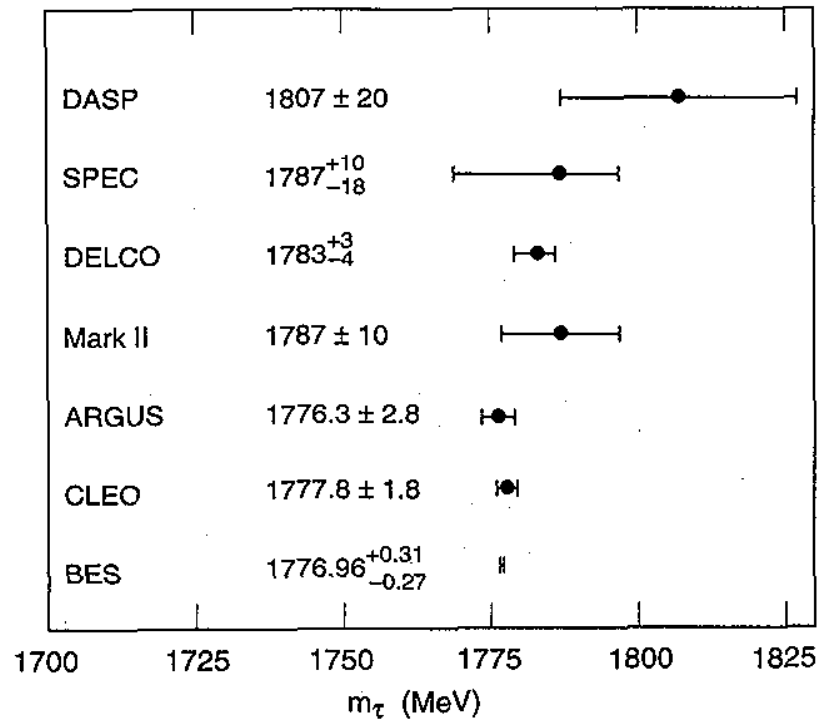

FIG. 20. A comparison of the value of the mass of the $\tau$ lepton obtained from the present analysis to the results from other experiments [2].

assuming $\tau \mu$ universality.

The point labeled HEP94 corresponds to the present values of $t_{\tau}$ and $B_{\tau \rightarrow e \nu \nu}( \pm 1 \sigma$ errors) [17], and shows the 1.3 standard deviation discrepancy indicated by Eq. (17). The dashed lines and point labeled PDG92 indicate the equivalent status using the 1992 Particle Data Group val-

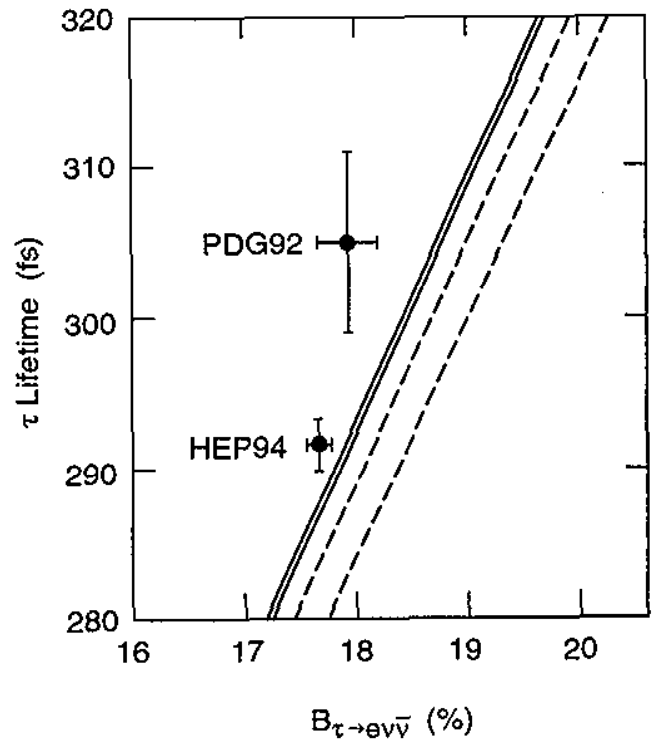

FIG. 21. The variation of the $\tau$ lepton lifetime $t_{\tau}$, with $B_{\tau \rightarrow e \nu \bar{\nu}}$ given by Eq. (6) under the assumption of lepton universality; the $\pm 1 \sigma$ bands obtained by using the value of $m_{\tau}$ from the present analysis (solid lines) and by using the PDG92 value [10] (dashed lines) are shown in comparison to the points ( $1 \sigma$ error bars) corresponding to the current average values of $t_{\tau}$ and $B_{\tau \rightarrow \text { e } \bar{\nu}}$ (HEP94 [17]) and the PDG92 values.
TABLE XI. The systematic error contributions to the uncertainty in the value of the mass of the $\tau$ lepton.

\begin{tabular}{lc}
\hline \hline Source & $\delta m_{\tau}(\mathrm{MeV})$ \\
\hline$\delta \epsilon= \pm 0.55 \%$ & +0.09 \\
$\delta \sigma_{B}=+0.74 \mathrm{pb}$ & +0.10 \\
Bias in the scanning procedure & \pm 0.10 \\
Uncertainty in the c.m. energy scale & \pm 0.09 \\
Uncertainty in the c.m. energy spread & \pm 0.02 \\
\hline Total systematic uncertainty & \pm 0.25 \\
\hline
\end{tabular}

ues [10]; these yield the value $0.9405_{-0.0242}^{+0.0250}$ for the coupling strength ratio. It follows that the deviation from $\tau \mu$ universality has decreased from 2.4 to 1.3 standard deviations as a result of the increased precision of the individual measurements.

The present experiment has reduced the uncertainty in the $m_{\tau}{ }^{5}$ factor in Eq. (6) to less than one part in 1000. However, in order to improve the overall precision of the squared coupling constant ratio of Eq. (17) to $\sim 2.5$ parts in 1000 (which would give the present discrepancy five standard deviation significance), $t_{\tau}$ would have to be known to within 0.4 fs and $B_{\tau \rightarrow e \nu \bar{\nu}}$ known to within $0.03 \%$. It does not appear likely that such precision will be attained in the near future, so that detecting the violation of $\tau \mu$ universality at the few percent level will remain a tantalizing possibility for some time to come [from Eq. (17), $3 \sigma \simeq 2.5 \%$ ].

\section{ACKNOWLEDGMENTS}

The BES Collaboration would like to thank the staffs of the BEPC accelerator and the Computing Center at the Institute of High Energy Physics, Beijing, for their outstanding efforts, and also Y. S. Tsai for helpful discussions about the $\tau^{+} \tau^{-}$cross section near threshold. The work of the BES Collaboration was supported in part by the National Natural Science Foundation of China under Contract No. 19290400 and the Chinese Academy of Sciences under Contract No. KJ85 (IHEP), by the Department of Energy under Contracts No. DE-FG02-91ER40676 (Boston University), DE-FG03-92ER40701 (California Institute of Technology), DE-FG03-93ER40788 (Colorado State University), DE-AC02-76ER03069 (MIT), DE-AC0376 SF00515 (SLAC), DE-FG03-91ER40679 (University of California at Irvine), DE-FG03-94ER40833 (University of Hawaii), DE-FG05-92ER40736 (University of Texas at Dallas), DE-AC35-89ER40486 (SSC Lab), by the U.S. National Science Foundation, Grant No. PHY9203212 (University of Washington), and by the Texas National Research Laboratory Commission under Contracts Nos. RGFY91B5, RGFY92B5 (Colorado State), and RCFY93-316H (University of Texas at Dallas). 
[1] W. J. Marciano and A. Sirlin, Phys. Rev. Lett. 61, 1815 (1988).

[2] Particle Data Group, L. Montanet et al., Phys. Rev. D 50, 1173 (1994).

[3] J. Z. Bai et al., Phys. Rev. Lett. 69, 3021 (1992).

[4] M. H. Ye and Z. P. Zheng, in Proceedings of the XIVth International Symposium on Lepton and Photon Interactions, Stanford, California, 1989, edited by M. Riordan (World Scientific, Singapore, 1990), p. 122.

[5] J. Z. Bai et al., Nucl. Instrum Methods A 344, 319 (1994).

[6] F. Porter (private communication); J. M. Wu and P. Y. Zhao, Chin. J. High Energy Nucl. Phys. 17, 379 (1993).

[7] É. A. Kuraev and V. S. Fadin, Yad. Fiz. 41, 733 (1985) [Sov. J. Nucl. Phys. 41, 466 (1985)].

[8] F. A. Berends and G. J. Komen, Phys. Lett. 63B, 432 (1976).

[9] M. B. Voloshin, in Proceedings of the $\tau$-Charm Factory Workshop, Stanford, California, 1989, edited by Lydia V. Beers (SLAC Report No. 343, Stanford, 1989).

[10] Particle Data Group, K. Hikasa et al., Phys. Rev. D 45,
S1 (1992).

[11] M. L. Perl et al., Phys. Rev. Lett. 35, 1489 (1975).

[12] The c.m. energy spread $\Delta$ is determined according to the equation $\Delta=(A \bar{I}+B)\left(C W^{2}+D\right)$, where $\tilde{I}$ is the average beam current; $A, B, C$, and $D$ are fitted to beam current measurements and to measurements of the energy spreads at the $J / \psi$ and $\psi^{\prime}$ resonances.

[13] S. Jadach and Z. Was, Comput. Phys. Commun. 64, 267 (1991).

[14] The acoplanarity angle $\theta_{\text {acop }}$ is defined as the angle between the planes spanned by the beam direction and the momentum vectors of the two final state charged tracks; i.e., it is the angle between the transverse momentum vectors of the two final state charged tracks.

[15] F. James and M. Roos, Comput. Phys. Commun. 10, 343 (1975).

[16] F. Porter, BES Report No. 93-013, 1993 (unpublished); L. Jones, Ph.D. thesis, Caltech, 1995.

[17] J. R. Patterson, in Proceedings of the XXVIIth International Conference on High Energy Physics, Glasgow, Scotland, 1994, edited by P. J. Bussey and I. G. Knowles (IOP, London, 1995), Vol. I, p. 149. 